\title{
Material herpetológico colectado por Roberto Donoso Barros en Venezuela (excepto geckos) en el Museo de Zoologia de la Universidad de Concepción, Chile
}

\author{
Herpetological material collected by Roberto Donoso Barros in Venezuela (except geckos) at \\ the Museum of Zoology, University of Concepción, Chile
}

\author{
César L. Barrio-Amorós ${ }^{1}$ \& Juan Carlos Ortiz ${ }^{2}$ \\ 1Doc Frog Expeditions, Uvita, Costa Rica. E-mail: cesarlba@yahoo.com \\ 2Departamento de Zoología, Universidad de Concepción, Chile. E mail jortiz@udec.cl
}

\begin{abstract}
RESUMEN
Se revisa el material herpetológico recolectado por Roberto Donoso Barros en Venezuela entre los años 1963 y 1964 , el cual se encuentra depositado en la colección de vertebrados de la Universidad de Concepción, Chile. Se ordena taxonómicamente respetando los conceptos actualmente establecidos. Por último, cuando es necesario se hacen comentarios de los ejemplares y especies con confusión taxonómica, haciendo énfasis en el material tipo descrito por este autor.
\end{abstract}

Palabras clave: Material tipo, Mannophryne riveroi, Pristimantis lancinii, Hyla robersimoni, Bachia marcelae.

\begin{abstract}
The material collected by Roberto Donoso Barros in Venezuela between 1963 and 1964 deposited at the Universidad de Concepción in Chile is catalogued and commented. Species are ordered following current systematic; specimens and species of interest are commented, especially those type material described by RDB.
\end{abstract}

Keywords: Type material, Mannophryne riveroi, Pristimantis lancinii, Hyla robersimoni, Bachia marcelae.

\section{INTRODUCCIÓN}

El herpetólogo chileno Roberto Donoso-Barros -RDB(1921-1975) pasó dos años en Venezuela entre 1963 y 1965, contratado por la Universidad de Oriente en Cumaná (Péfaur 1976), realizando extensas colecciones de reptiles y anfibios tanto en el área donde trabajó (oriente de Venezuela), como en el resto del país. Con este material, describió algunos reptiles y anfibios nuevos, como los anuros Prostherapis riveroi $=$ actualmente Mannophryne riveroi (Donoso-Barros 1964), Eleutherodactylus lancinii $=$ ahora Pristimantis lancini (Donoso Barros 1968) y los reptiles Bachia marcelae (hoy una subespecie de Bachia heteropa, aunque el estado taxonómico debe revisarse), y los geckos diurnos del género Gonatodes (G. ceciliae, G. ligiae, G. petersi y G. seiglei). En este trabajo revisamos y se emiten comentarios sobre la colección herpetológica que reposa en la Universidad de Concepción, Chile (excepto los geckos -familias Gekkonidae, Phyllodactylidae y Sphaerodactylidae- que son tratados en otro trabajo aparte). Para cada especie recolectada por R. Donoso Barros en
Venezuela se ofrecen los números actuales asignados en el MZUC (Museo de Zoología de la Universidad de Concepción, Concepción, Chile), el sexo de cada individuo (cuando es posible sexarlo), la fecha y la localidad de recolección, y comentarios cuando se precisa. Se sigue para anfibios la clasificación de Frost (2014) y para reptiles la de Rivas et al. (2012). El orden en que se entregan es alfabético y los cambios nomenclaturales que haya habido desde entonces son, comentados en cada especie. En las especies que poseen más de un autor, los autores se separan con la conjunción latina "et" que significa " $y$ " en lugar del símbolo "\&".

\section{AMPHIBIA}

Familia Bufonidae

Atelopus cruciger (Lichtenstein et Martens, 1856). Lámina 1A Material: MZUC 8580, 37603-09, 37611, 37613, 37615616 (machos), 37610, 37612, 37614, 37617, 37618-19 (juvs), 14 febrero 1964, Rancho Grande, Parque Nacional Henri Pittier, Estado Aragua. 
Comentario: En la época en que RDB visitó Rancho Grande (1964), esta especie era aún abundante. Actualmente (aparentemente después de 1986, Bonnacorso et al. 2003) se considera extinguida de la mayoría de poblaciones conocidas históricamente, solo sobreviviendo en 3 localidades de zonas bajas, siendo monitoreadas en la actualidad (Lampo et al. 2011).

Atelopus mucubajiensis Rivero, 1972. Lámina 1B Material: MZUC 8539 (hembra adulta), 37601 (subadulto), 37602 (juv), 22 marzo 1964, Apartaderos, sierra de Santo Domingo, Estado Mérida.

Comentario: Esta especie se considera extinta en la naturaleza (Barrio-Amorós 2004a; datos propios).

Rhinella humboldti (Gallardo, 1965). Lámina 1C

Material: MZUC 11294 (hembra adulta), sin fecha, El Tukuko, Perijá, Estado Zulia. MZUC 11293, 34169-70, 34172-73 (machos), 34171 (hembra), 25 junio 1963, Guanare, Estado Portuguesa.

Comentario: Catalogado como Bufo granulosus. Narvaes y Rodrigues (2009) elevan Rhinella granulosa humboldti a estatus específico.

Rhinella marina (Linnaeus, 1758). Lámina 1D

Material: MZUC 11628 (hembra adulta), 30 mayo 1963, Macuro, Estado Sucre. MZUC 11228 (juv), 10 abril 1963, Caripito, Estado Monagas.

\section{Familia Dendrobatidae}

Aromobates leopardalis Rivero, 1976

Material: MZUC 41221 (macho adulto), 8567 y 41220 (hembras adultas), 41222 (subadulto), MZUC 8568, 4122528 (no sexados), 22 de marzo 1964, Santo Domingo, Estado Mérida, Venezuela

Comentario: Introducidos en el catálogo como Prostherapis collaris.

\section{Aromobates sp.}

Material: MZUC 8593, MZUC 41229-39, 9 diciembre, El Mirador San Cristóbal, Estado de Táchira.

Comentario: Introducidos en el catálogo como Colostethus alboguttatus.

Mannophryne collaris (Boulenger, 1912). Lámina 1E Material: MZUC41268 (macho), 41267 (hembra), 8592, 41266 (subadultos), 41269 (juv), 41265 (larva), 11 diciembre 1963, rio Mocotíes, Estado Mérida.

Comentario: como $M$. collaris fueron identificadas casi todas las especies acollaradas andinas. La Marca (1994) y Barrio-Amorós et al. (2010a) aclaran la situación sistemática del género en los Andes venezolanos. Barrio-Amorós et al. (2010a) restringen la distribución de $M$. collaris a las cuencas de los ríos Chama y Mocotíes (estado Mérida).
Mannophryne aff. herminae (Boettger, 1893). Lámina 1F Material: MZUC 11039, 41200, 41207, 41210-11 (hembras), 8 septiembre 1963, Rancho Grande, Parque Nacional Henri Pittier, Estado Aragua.

Comentario: Introducidos en la colección como Prostherapis trinitatis. Consideramos la población de Mannophryne de Rancho Grande como aff. herminae hasta que ulteriores estudios aclaren la situación taxonómica. Barrio-Amorós et al. (2006, 2010a) aclaran la situación de M. trinitatis en Venezuela.

Mannophryne riveroi Donoso Barros, 1964. Figuras 1 y 2 Material: MZUC 8566 (holotipo; V 307 en la publicación original, supuestamente macho adulto), 30 abril 1964, Cerro Azul, Macuro, Estado Sucre. En la descripción original se mencionan dos machos paratipos con los números V $306 \mathrm{y}$ 308 , respectivamente MZUC 12044 y 12045 . Se mencionan dos hembras paratipicas 309 y 310, de las cuales sólo hemos hallado en la colección a 310 , que actualmente es MZUC 12046.

Medidas del holotipo: Las medidas del holotipo son las siguientes: Longitud Cabeza Cuerpo: 40.1; Longitud de la Tibia: 19.4; Longitud del Muslo: 18.3; Longitud del pie: 19.8; Longitud de la mano: 12.1; Ancho de la Cabeza: 16.2; Largo de la Cabeza: 14.6; Distancia internasal: 5.2; Distancia entre la comisura anterior del ojo y el centro de la narina: 3.7; Longitud del hocico desde el borde anterior del ojo hasta la punta del hocico: 5.8; Diámetro horizontal del ojo: 5.2; Diámetro horizontal del tímpano: 3.1; Anchura del disco del tercer dedo: 1.4; Anchura del disco del cuarto dedo del pie: 1.6; Longitud del primer dedo de la mano, desde el borde interno del tubérculo tenar hasta la punta del disco: 7.2; Longitud del segundo dedo de la mano desde la intersección de los dedos I y II hasta la punta del disco: 7.0 (medidas tomadas siguiendo a Barrio-Amorós et al. 2010a). Comentario: Descrito originalmente como Prostherapis riveroi. El sexo del holotipo no ha podido ser verificado dado que su parte ventral se encuentra deteriorada y no hemos querido abrir más para no dañar el espécimen, que no se encuentra en las mejores condiciones. No obstante, basados en la coloración del ejemplar, es más probable que se trate de una hembra (comparar Fig 1 con Fig 2). También la coloración que describe Donoso Barros (1964) corresponde a una hembra (ver Barrio-Amorós et al. 2010a), pero dado el estado actual del holotipo es difícil de determinar.

La medida que Donoso Barros (1964) da del holotipo es de $44 \mathrm{~mm}$, cuando nuestra propia medida es de $40.1 \mathrm{~mm}$; eso puede deberse fácilmente a la deshidratación post preservación que ocurre a muchos especímenes.

Barrio-Amorós et al. (2010b) encuentran una alta incidencia de malformaciones en dedos de manos y pies en una serie colectada en 2001. Revisando la serie típica, solo hemos hallado una anomalía: en MZUC 12046 el dedo IV de la mano derecha es más corto que el dedo II, faltándole una falange pero con el disco terminal completo. 


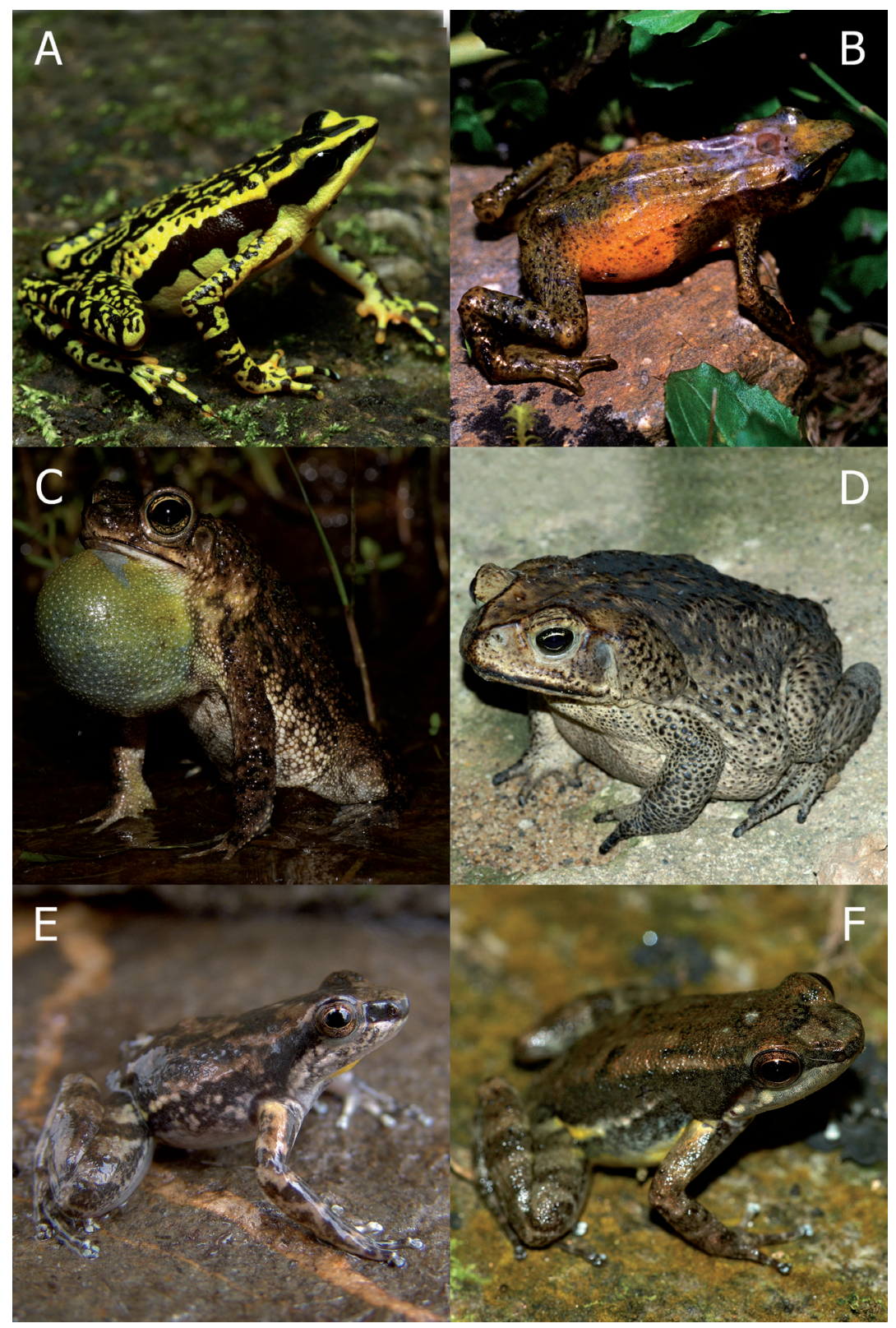

LÁmina 1. A. Atelopus cruciger: Una de las especies más amenazadas de Venezuela. La población donde sobrevive esta especie no puede ser mencionada. B. Atelopus mucubajiensis: El ejemplar hembra de la foto corresponde al último conocido en vida, conseguido en septiembre de 2004. Se teme que haya engrosado las filas de las especies extinguidas. C: Rhinella humboldti es una especie del complejo de $R$. granulosa, ampliamente repartido por Suramérica; Puerto Ayacucho, Estado Amazonas. D: Rhinella marina, este es uno de los sapos de mayor tamaño y más ampliamente distribuidos del Neotropico; La Acequia, Estado Barinas. E: Mannophryne collaris. Antaño abundante en todo el valle del río Chama, donde se sitúa la ciudad de Mérida, la progresiva extensión de la ciudad y los cultivos circundantes han llevado a esta especie a sobrevivir exclusivamente en lugares prístinos; Los Ranchos, Santa Cruz de Mora, Estado Mérida. F: Mannophryne herminae. Donoso Barros visitó Rancho Grande, en el Estado Aragua, una de las estaciones biológicas más conocidas de Venezuela, donde colectó esta especie común en sus quebradas.

Plate 1. A. Atelopus cruciger: One of the most threatened species in Venezuela. The population where it survives cannot be mentioned. B. Atelopus mucubajiensis: The female specimen in the picture correspond to the last known in life, found in September 2004. It is feared to engrossed the extinct numbers of extinct species. C: Rhinella humboldti is a species in the R. granulosa Group, widely spread through South America east of the Andes; Puerto Ayacucho, Estado Amazonas. D: Rhinella marina, this is one of the largest anurans and widely spread in the Neotropics; La Acequia, Estado Barinas. E: Mannophryne collaris, formerly abundant in the entire Chama river valley, where the city of Mérida is sited: the progressive grown of the city and crop fields around, lead this species to survive only in rare pristine places. Los Ranchos, Santa Cruz de Mora, Estado Mérida. F: Mannophryne herminae. Donoso Barros visited Rancho Grande in Aragua state, one of the best known biological stations of the country, where he collected this common species in the surrounding creeks. 


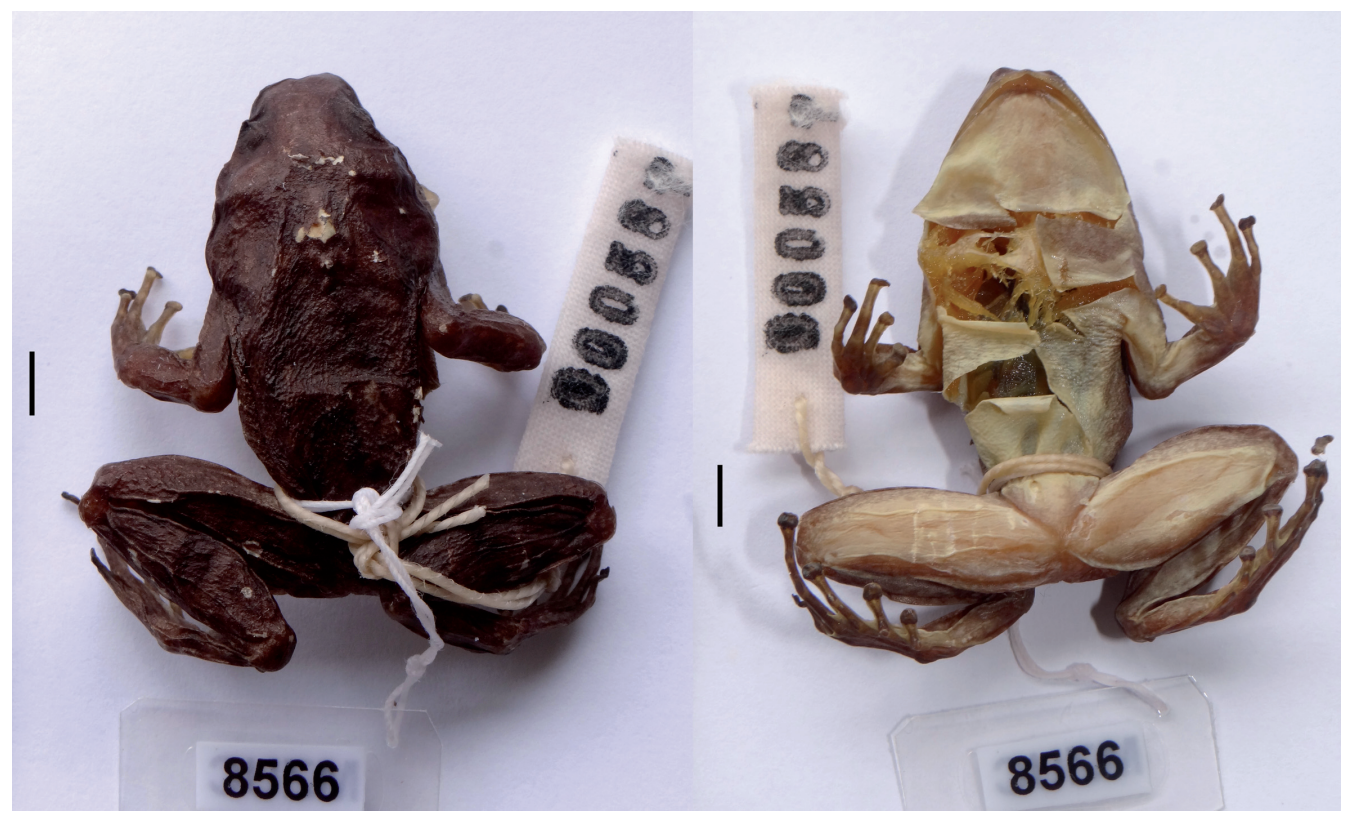

FIgURA 1. Vistas dorsal y ventral del holotipo de Prostherapis riveroi (MZUC 8566), actualmente Mannophryne riveroi. Fotos: C.L. BarrioAmorós.

FIGURE 1. Prostherapis riveroi (MZUC 8566), holotype in dorsal and ventral views, actually Mannophryne riveroi. Photos: C. L. BarrioAmoros.

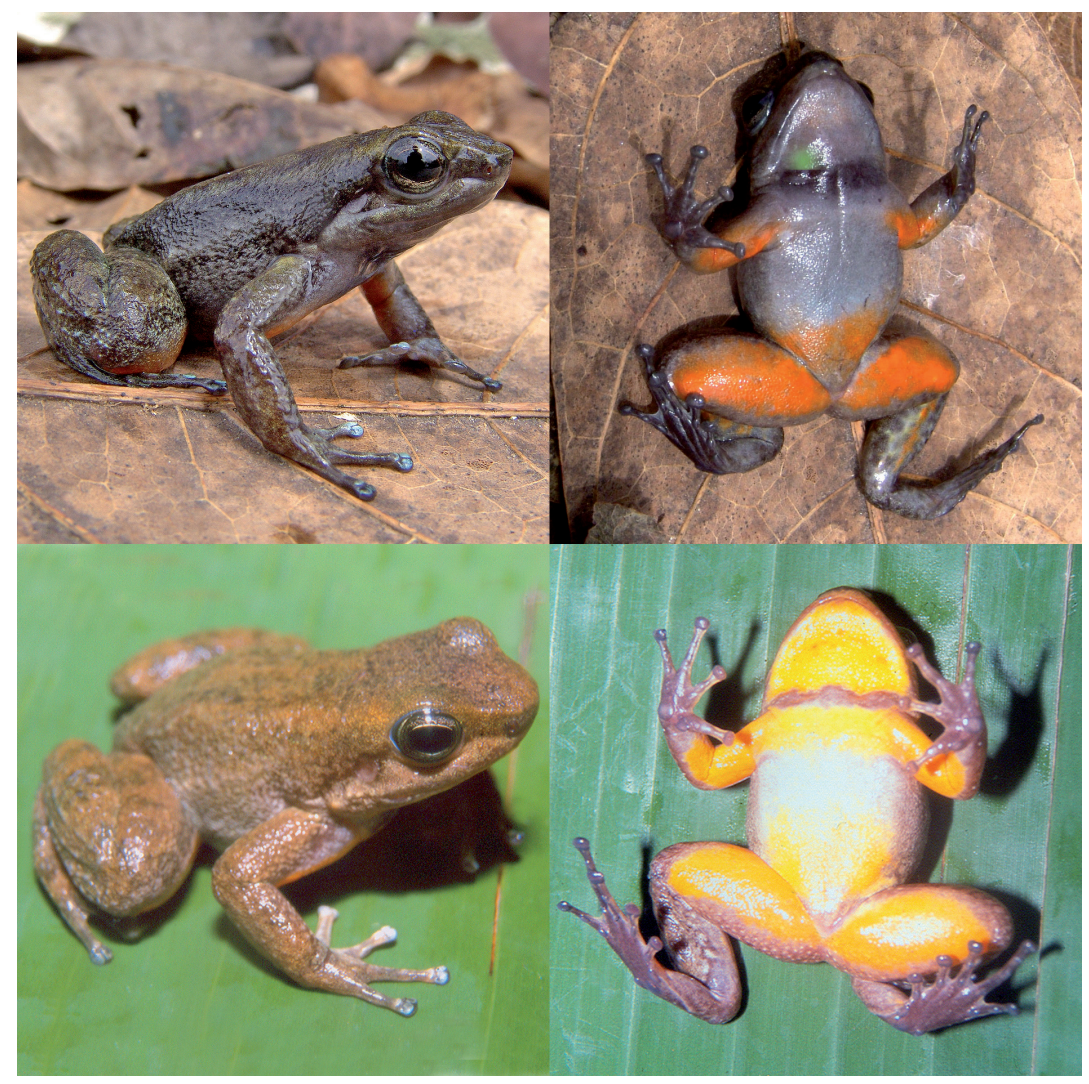

Figura 2. Mannophryne riveroi en vida, de Las Melenas, Península de Paria, Estado Sucre. Arriba macho; abajo hembra. Fotos: C.L. Barrio-Amorós.

Figure 2. Mannophryne riveroi inlive, Las Melenas, Paria Peninsula, Sucre State. Up, male; down, female. Photos: C. L. Barrio-Amoros. 
Mannophryne venezuelensis Manzanilla, Jowers, La Marca et García-Paris, 2007. Lámina 2A

Material: MZUC 41219 (macho); 8594, 41212-15, 4121718 (hembras), 6 julio 1963, quebrada Guaraguara, cerca de Güiria, Estado Sucre.

Comentario: Ingresados en la colección como Prostherapis trinitatis. Barrio-Amorós et al. (2006) y Manzanilla et al. (2007) reasignan las poblaciones de $M$. trinitatis venezolanas a otras especies. La población de "M. trinitatis" de la península de Paria que colectó RDB es actualmente $M$. venezuelensis. Mannophryne trinitatis es endémico de la isla de Trinidad.

\section{Familia Hemiphractidae}

Flectonotus pygmaeus (Boettger, 1893). Lámina 2B

Material: MZUC 11290 (hembra), 16 marzo, 1964, Rancho Grande, Parque Nacional Henri Pittier, Estado Aragua.

\section{Familia Hylidae}

Dendropsophus meridensis (Rivero, 1961). Lámina 2C Material: MZUC 40158 (hembra adulta), marzo 1964, cerca de Mérida, Estado Mérida.

Comentario: ingresado a la colección como Hyla labialis.

Dendropsophus microcephalus (Cope, 1886). Lámina 2D Material: MZUC 8603, 8531, 8534, 41187, 12 agosto 1964, Tucupita, Estado Delta Amacuro. MZUC 11232, 40188 (macho adulto), agosto 1963, La Rinconada, Cumanacoa, Estado Sucre. MZUC 11231, 40205-208, 40210-213 (machos adultos), 40209 (hembra adulta), julio 1963, Santa Fe, Estado Sucre. MZUC 11233 (macho), 10 octubre 1963, Playa Colorada, Estado Sucre. MZUC 8624, 40194-201 (machos), sin fecha, El Gateado, Barinitas, Estado Barinas. Comentario: Algún material está demasiado deshidratado para poder sexarlo. Algunos ejemplares fueron catalogados como Hyla riveroi o H. minuta por RDB. Actualmente todos pertenecen al taxón Dendropsophus microcephalus.

Hypsiboas crepitans (Wied-Neuwied, 1824). Lámina 2E Material: MZUC 8514, septiembre 1960, Caracas, Distrito Federal (Leg. J. Roze). MZUC 11242 (macho adulto), julio 1963, Cumaná, Estado Sucre. MZUC 40220-21 (machos adultos), 8522-23 (hembras adultas), septiembre 1963, Playa Colorada, Estado Sucre. MZUC 8640, 41179-81 (macho adulto), 22 octubre 1963, Playa Colorada, Estado Sucre. MZUC 40219 (macho adulto) septiembre de 1963, Playa Colorada Estado de Sucre. MZUC 8569 (juvenil) 26 de agosto 1963, Playa Colorada, Estado de Sucre. MZUC 8641 (macho adulto), 9 diciembre 1963, La Tendida, Estado Táchira.

Phyllomedusa trinitatis Mertens, 1926. Lámina 2F

Material: MZUC 11248 (macho), 22 octubre 1963, Playa Colorada, Estado Sucre. MZUC 11291 (macho), 29 mayo 1964, Macuro, Península de Paria, Estado Sucre.
Material: MZUC 11638 (hembra), 3 junio 1964, Playa Colorada, Estado Sucre. MZUC 11245 (hembra adulta), 6 abril, 1964, San Bonifacio, Estado Monagas. MZUC 8530 (macho), sin fecha, Caripito, Estado Monagas. MZUC 11196, 40230, 40232 (machos adultos), 40231 (hembra adulta), sin fecha, San Bonifacio, Estado Monagas.

Comentario: Ejemplares de esta especie fueron identificados como Hyla foliamorta (=sinónimo actualmente de $S$. rostratus desde Rivero 1968) por RDB.

Scinax ruber (Laurenti, 1768). Figura 3

Material: MZUC 8544 (macho holotipo de Hyla robersimoni), antes RBD 527; 30 mayo 1964, "Pajonales de la Península de Paria”, Macuro, Estado Sucre. MZUC 12035, 36 (machos paratipos de Hyla robersimoni), 30 mayo 1964, misma data que holotipo. MZUC 11292 (hembra), 40187 (macho) 10 abril 1964, Caripito, Estado Monagas. MZUC 8533 (macho), 8602, 40185-86 (ejemplares deshidratados que no se pueden sexar), 12 agosto 1964, Tucupita, Estado Delta Amacuro. MZUC 11637 (hembra), 3 junio 1964, Playa Colorada, Estado Sucre.

Comentario: Hyla robersimoni Donoso Barros, 1965 fue sinonimizada con Scinax ruber por Duellman (1977). La Fig 3A ilustra el holotipo de Hyla robersimoni. Fig 3B muestra un macho de Scinax ruber en vida.

Scinax x-signatus (Spix, 1824). Lámina 3B

Material: MZUC 8532 (juv), 8 noviembre 1963, El Limón, Estado Aragua.

Comentario: Identificado como Hyla (=Scinax) baumgardneri por RDB. El nombre $x$-signatus esconde un complejo de especies (Barrio-Amorós 2004b).

Trachycephalus typhonius (Linnaeus, 1758). Lámina 3C Material: MZUC 11179 (hembra adulta), 7 julio 1963, cerca de Güiria, Estado Sucre.

Comentarios: Esta especie ha sufrido varios cambios nomenclaturales en los últimos tiempos. Conocido hasta la revisión filogenética de Faivovich et al. (2005) como Phrynohyas venulosa, cuando se traspasa al género Trachycephalus. Más recientemente Lavilla et al. (2011) resuelven un problema nomenclatural antiguo asignando el nomen typhonius a Trachycephalus venulosus; así pues, actualmente T. typhonius.

\section{Familia Leptodactylidae}

Leptodactylus insularum Barbour, 1906. Lámina 3D

Material. MZUC 8880, (hembra), MZUC 40202-04, 4021718 (ejemplares en muy mal estado), julio 1963, Cumaná, Estado Sucre. MZUC 11282 (macho subadulto), 10 diciembre 1963, Valera, Estado Trujillo.

Comentario: Catalogados en la colección como L. bolivianus. Heyer y de Sá (2011) demuestran que las poblaciones del norte de Venezuela pertenecen a $L$. insularum. 


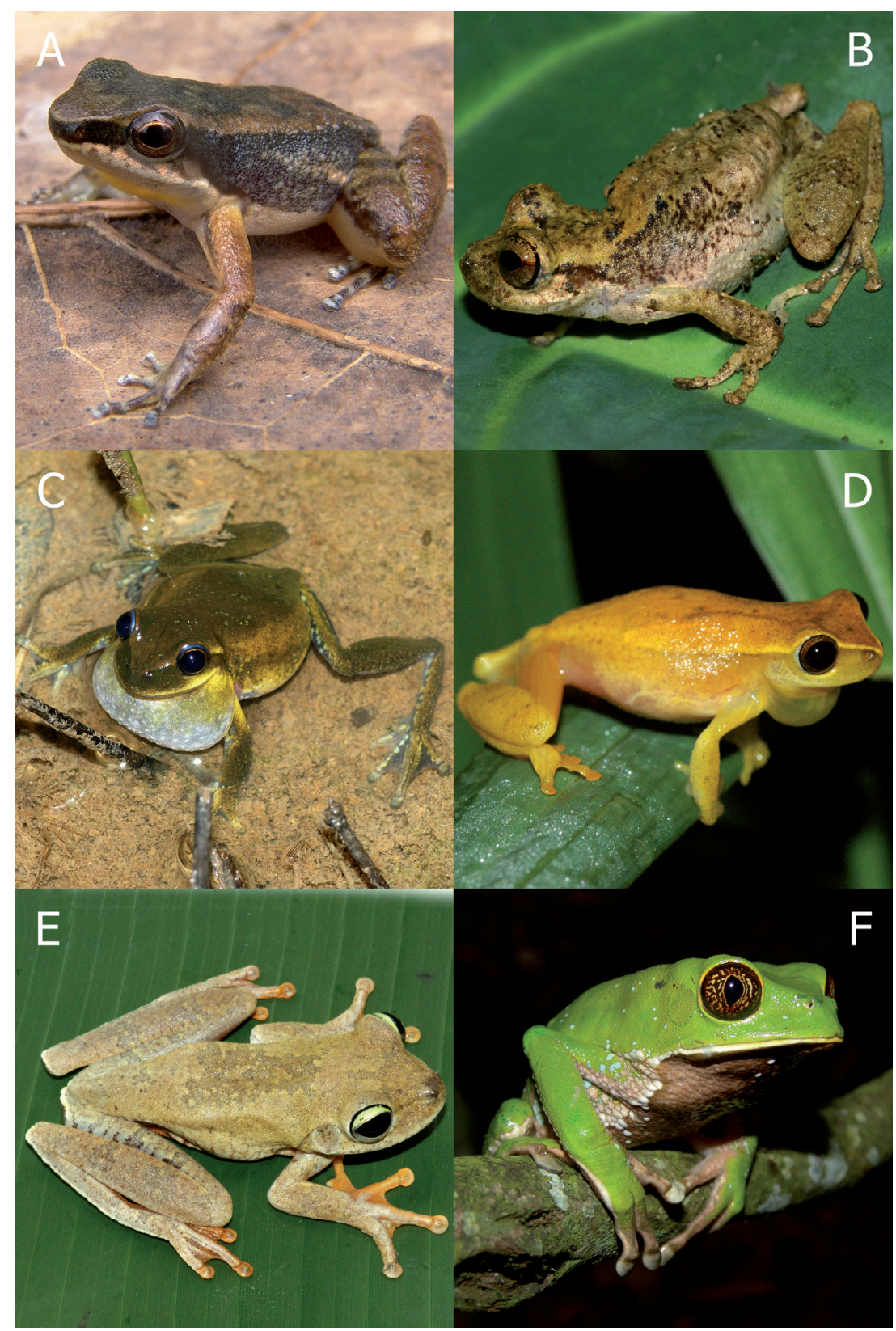

LÁmina 2. A: Mannophryne venezuelensis. Especie de reciente descripción, confundida por Donoso y otros autores con M. trinitatis; Las Melenas, Península de Paria, Estado Sucre. B: Flectonotus pygmaeus. Esta pequeña especie de hemifráctido o ranita marsupial, es común en Rancho Grande, Estado Aragua. C: Dendropsophus meridensis. Una rana endémica de la Cordillera de Mérida, donde se ha enrarecido especialmente donde se ha introducido a la rana toro Lithobates catesbeianus; La Carbonera, Estado Mérida. D: Dendropsophus microcephalus. Una de las especies más abundantes e omnipresentes en zonas bajas abiertas. Chururú, Estado Táchira. E: Hypsiboas crepitans. Otra especie ubicua y muy antropófila, hallándose en ciudades y en zonas rurales, siempre asociada a áreas abiertas. Caracas, Distrito Federal. F: Phyllomedusa trinitatis. Sin duda una de las especies más hermosas de hílidos. Ubicua en zonas bajas y de mediana altitud en la Cordillera de la Costa. Caracas, Distrito Federal.

Plate 2. A: Mannophryne venezuelensis. Species of recent description, confounded by Donoso and other authors with M. trinitatis; Las Melenas, Peninsula de Paria, Estado Sucre. B: Flectonotus pygmaeus, this small marsupial frog is common in Rancho Grande, Aragua state. C: Dendropsophus meridensis, an endemic from the Cordillera de Mérida, where it has become pretty rare due to the introduction and proliferation of the bullfrog Lithobates catesbeianus; La Carbonera, Estado Mérida. D: Dendropsophus microcephalus. Una de las especies más abundantes e omnipresentes en zonas bajas abiertas. Chururú, Estado Táchira. E: Hypsiboas crepitans, another ubiquitous species, very anthropophilous, being found in rural areas as well as city gardens, in nature always in open areas; Caracas, Distrito Federal. F: Phyllomedusa trinitatis, with no doubt one of the most beautiful species of hylids in northern Venezuela. It is ubiquitous in low and uplands through the Cordillera de la Costa. Caracas, Distrito Federal. 
Leptodactylus macrosternum Miranda-Ribeiro, 1926. Lámina 3E

Material: MZUC 11296 (hembra), 10 octubre 1964, cerca de Macuro, Estado Sucre. MZUC 11295, 40181-82 (hembras), junio 1964, Valle de la Pascua, Estado Guárico.

Comentario: Catalogado como Leptodactylus ocellatus.

Leptodactylus cf. petersi (Steindachner, 1864). Lámina 3F Material: MZUC 40156, 40165 (machos), MZUC 12283 (hembra), 10 abril 1964, Cueva del Guácharo, Estado Monagas.

Comentario: La taxonomía del grupo de Leptodactylus del grupo podicipinus-wagneri no está resuelta, y es compleja (Heyer 1994).

Leptodactylus turimiquensis Heyer, 2005. Lámina 4A

Material: MZUC 11129, 40162 (machos adultos), 12 junio 1963, Estado Sucre.

Comentarios: Heyer (2005) describe L. turimiquensis con base a los "L. labyrinthicus" del NE de Venezuela. Los especímenes colectados por RBD fueron determinados como L. pentadactylus. Aunque la localidad "Sucre" comprende todo el estado del mismo nombre, dado que RDB había colectado extensamente en la Península de Paria, donde esta especie se distribuye principalmente, pensamos que los especímenes provienen de dicha área.

Leptodactylus sp. Lámina 4B

Material: MZUC 40172, 40174 (machos adultos), MZUC
8591, 40173, 40175 (hembras), 22 marzo 1964, Rio Chama, Estado Mérida.

Comentario: Esta especie fue catalogada como Leptodactylus podicipinus petersi por RDB. R. Heyer (com. pers.) opina que se trata de una nueva especie no descrita del grupo podicipinus-wagneri.

\section{Familia Microhylidae}

Elachistocleis ovalis (Schneider, 1799). Lámina 4C

Material: MZUC 12289, 33644-46 (subadultos), noviembre 1964, El Limón, Estado Aragua.

\section{Familia Pipidae}

Pipa parva Ruthven et Gaige, 1923. Lámina 4D

Material: MZUC 8570, 41585-615 (juvs), 26 marzo 1964, cerca de Tukuko, Sierra de Perijá, Estado Zulia.

\section{Familia Strabomantidae}

Pristimantis ginesi (Rivero, 1964). Lámina 4E

Material: MZUC 11155, 2 febrero, 1963, Sierra Santo Domingo, Estado Mérida.

Comentario: este ejemplar es considerado topoparatipo (=paratopotipo) en las notas del catálogo de RDB, pero Rivero (1964) solo menciona un holotipo en la serie típica. Ignoramos si RDB pretendía que su espécimen fuera considerado parte de la serie tipo, pero en dado caso eso no sucedió.

Pristimantis lancinii (Donoso Barros, 1965). Figura 4 Material: Los números que aparecen en la publicación
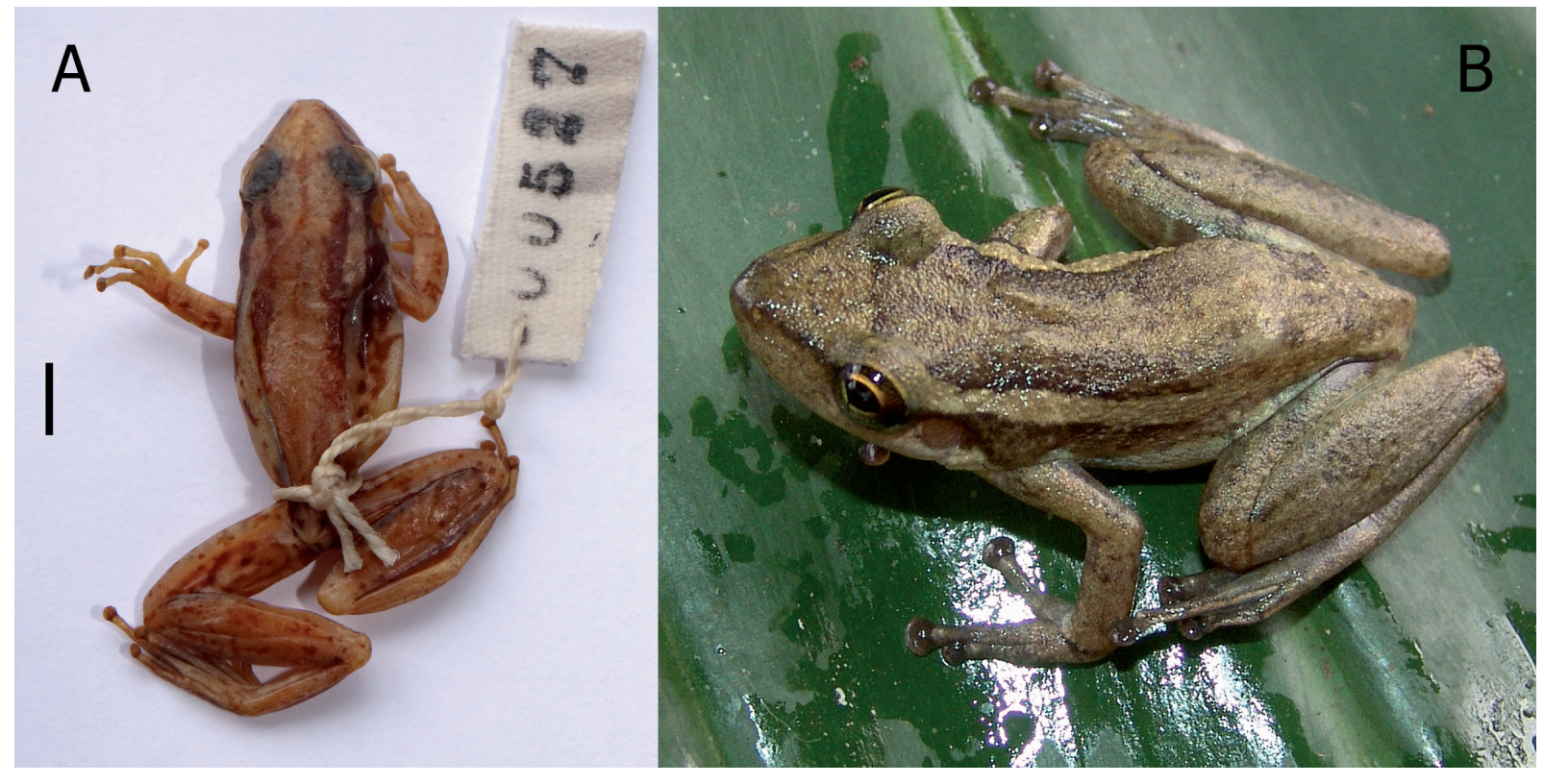

Figura 3. 3A Holotipo de Hyla robersimoni (MZUC 8544). 3B Scinax ruber macho vivo en coloración diurna.

FIgURE 3. 3A Holotype of Hyla robersimoni (MZUC 8544). 3B Scinax ruber, inlive male in day color. 


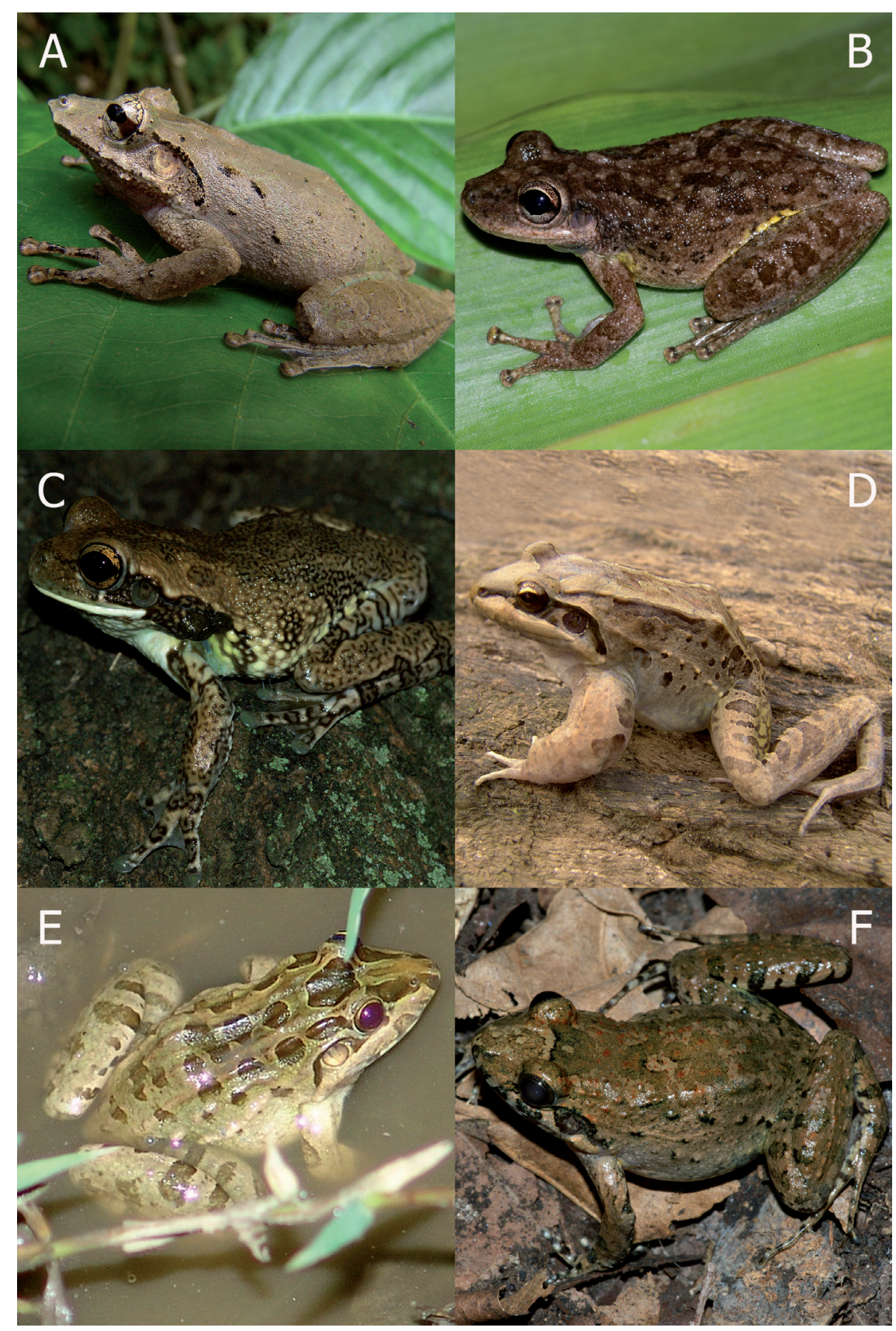

LÁmina 3. A: Scinax rostratus. Especie bien distribuida por el norte de Suramérica, pero poco frecuente. A veces se agrupan en frenética actividad reproductiva. Bejuma, Estado Carabobo. B: Scinax x-signatus. Sin duda un nombre que enmaraña un complejo de especies a resolver. Caracas, Distrito Federal. C: Trachycephalus typhonius. Especie también bastante antropófila, pero rara en la naturaleza. Puede secretar una sustancia lechosa y pegajosa por su piel. D: Leptodactylus insularum. Conocido hasta hace poco como L. bolivianus, esta especie grande se distribuye por todo el norte del país. Río Paguey, Estado Barinas. E: Leptodactylus macrosternum. Especie muy abundante pero restringida a los llanos de Venezuela y Colombia. Río Paguey, Estado Barinas. F: Leptodactylus petersi. Una especie también común pero de un complejo taxonómico aun no resuelto. Caracas, Distrito federal.

Plate 3. A: Scinax rostratus, well distributed species through northern South America, but not frequent; sometimes they gather in frenetic reproductive activity. Bejuma, Estado Carabobo. B: Scinax x-signatus, with no doubt a name that hides a species complex to be solved. Caracas, Distrito Federal. C: Trachycephalus typhonius. Another usually anthropophilous species, but rare in nature. It can secrete a milky sticky glue to be avoided; Imataca, Estado Bolivar. D: Leptodactylus insularum, known until recently as L. bolivianus, this large species is distributed along all northern Venezuela; Río Paguey, Estado Barinas. E: Leptodactylus macrosternum, abundant species but restricted to open áreas of Venezuela and Colombia; Río Paguey, Estado Barinas. F: Leptodactylus petersi, a common and small species pertaining to a species complex to be taxonomically solved. Caracas, Distrito federal. 


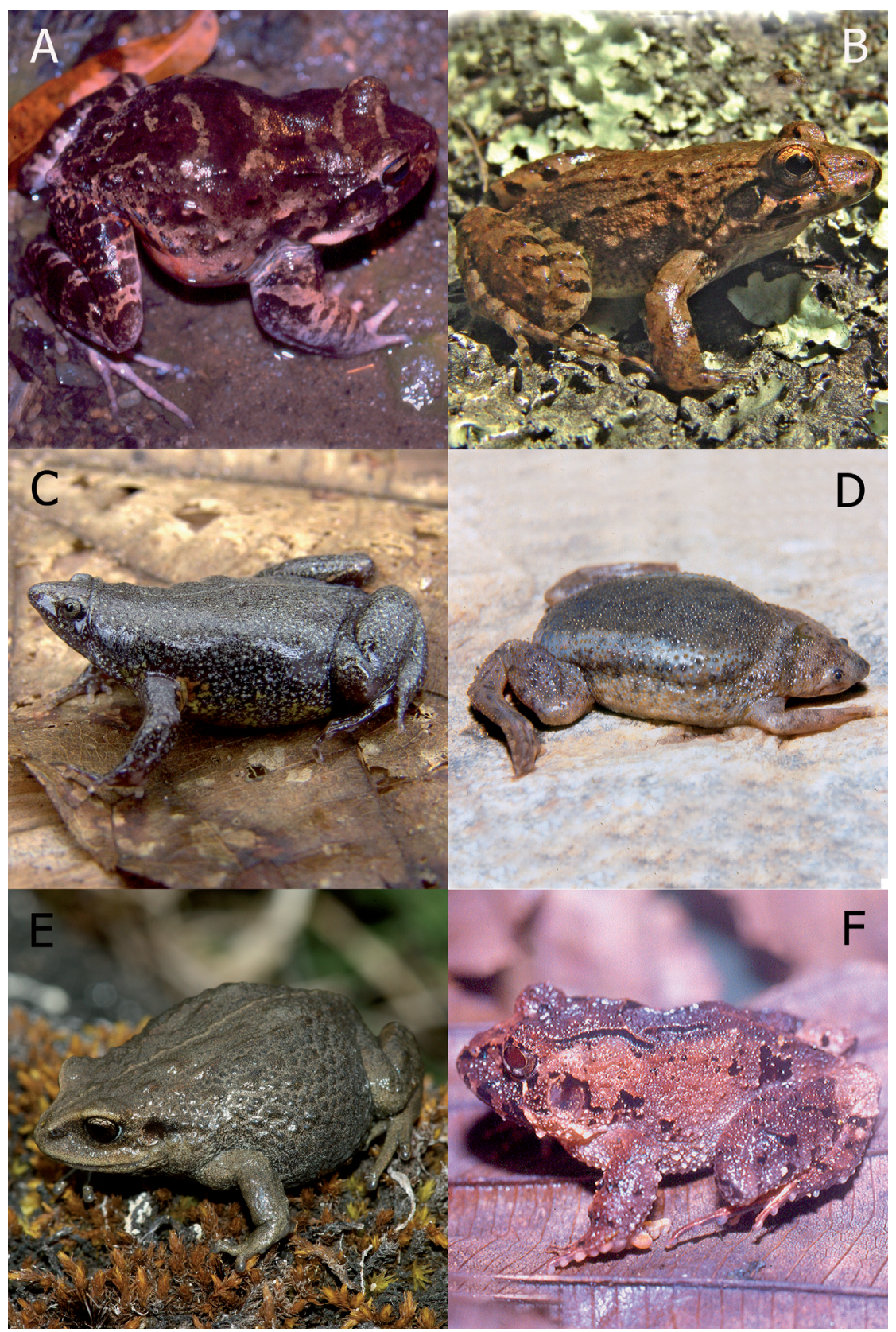

LÁmina 4. A: Leptodactylus turimiquensis. Especie de gran tamaño que habita solamente el sector NE de Venezuela. Las Melenas, Estado Sucre. B: Leptodactylus sp. Especie no descrita habitante de varios valles andinos; Lagunillas, Estado Mérida. C: Elachistocleis ovalis. Microhílido que se mantiene enterrado la mayor parte del año, alimentándose de termitas subterráneas, aparece durante las primeras semanas de las lluvias a reproducirse frenéticamente. Chururú, Estado Táchira. D: Pipa parva. Rana de forma aplanada, y completamente acuática. Las hembras guardan sus huevos en un substrato dorsal donde se desarrollan, y aparecen los renacuajos al tiempo para terminar su desarrollo en el agua. El Vigía, Estado Mérida. E: Pristimantis ginesi. La especie que habita a mayor altitud en el país entre 3000 y 4400 msnm; Valle de Sai sai, Estado Mérida. F: Strabomantis biporcatus. La rana de desarrollo directo más grande del país; era común en Rancho Grande, Estado Aragua, pero se ha rarificado mucho en la última década.

Plate 4. A: Leptodactylus turimiquensis, large species endemic to NE Venezuela; Las Melenas, Estado Sucre. B: Leptodactylus sp. An still not described species occurring at different Andean valleys. Lagunillas, Estado Mérida. C: Elachistocleis ovalis, a microhylid that lives under ground around the year, feeding on subterranean termites. It appears in large numbers after the first rains to reproduce frantically. Chururú, Estado Táchira. D: Pipa parva. Strange looking frog, completely aquatic; females keep its eggs inside a dorsal foam-like substrate, where the tadpoles appear to finish its development in the water. El Vigía, Estado Mérida. E: Pristimantis ginesi. This is the species that can survive at a higher altitude in the country, reaching $4000 \mathrm{~m}$ asl.; Valle de Sai sai, Estado Mérida. F: Strabomantis biporcatus. This is the largest Terrarana in the country, or direct developer; it was common at Rancho Grande, but it has become quite rare nowadays. 
original corresponden a la libreta de campo de RDB, por lo que los números finales se relatan a continuación: MZUC 8538 (=511, hembra adulta), 8524 (=491), 12246 (=492), 12247 (=493), 12248 (=494), 12249 (=495), todos juveniles o subadultos. 19 marzo 1963, Páramo de Apartaderos, Estado Mérida. Todos son considerados paratipos.

Comentarios: RDB 490 no aparece en la colección de MZUC. Frost (2014) establece que el holotipo de esta especie está en el USMN (como 165604, ver Fig 4). Frost (2014) se equivoca en varios aspectos de sus comentarios. El número original del holotipo es 00490, no 40490. Por otro lado, la especie nunca fue descrita como Eupsophus lancinii, sino como Eleutherodactylus lancinii, tanto en
Donoso Barros (1965) como en Donoso Barros (1968). Solo en la Fig de la pag 3 de la descripción original aparece como Eupsophus lancinii.

Strabomantis biporcatus Peters, 1863. Lámina 4F Material: MZUC 40225 (hembra adulta), 8546, 40223-24, 40226-27 (juveniles), 20 octubre 1963, Rancho Grande, Parque Nacional Henri Pittier, Estado Aragua.

Comentario: Ingresados a la colección como Eleutherodactylus cornutus maussi, esta especie ha sufrido constantes cambios nomenclaturales (Barrio-Amorós y Kaiser 2008) hasta su posición final (igual a la original), Strabomantis biporcatus.

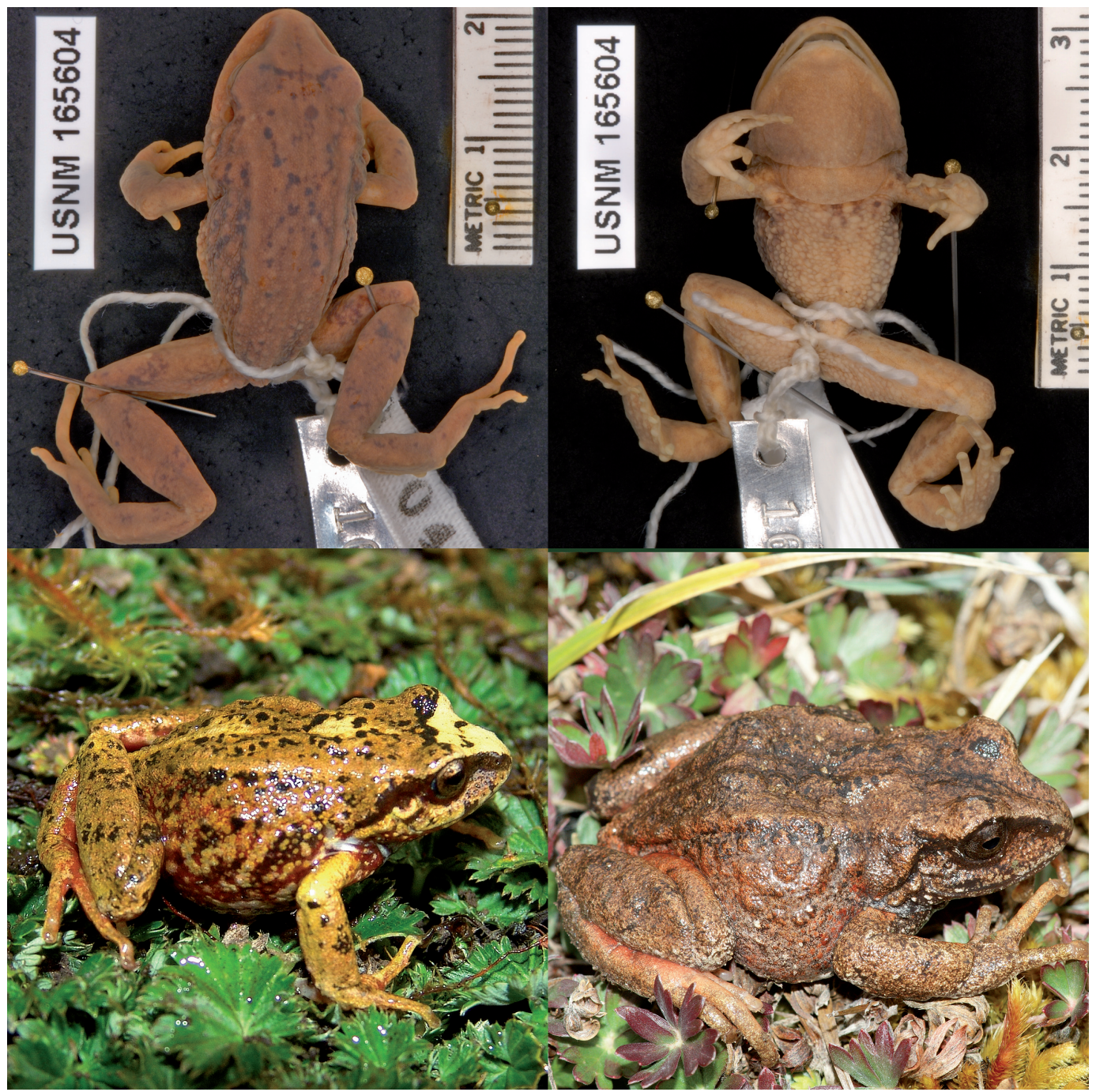

Figura 4. Pristimantis lancinii. Arriba vistas dorsal y ventral del holotipo colectado por RDB (USNM 165604), actualmente en el United States National Museum, Smithsonian Institution, Washington DC. Abajo dos cromotipos de la especie procedente de la Quebrada Corcovada, Estado Mérida, muy cerca de la localidad tipo.

Figure 4. Pristimantis lancini. Up, holotype in dorsal and ventral views collected by RDB (USNM 165604), actually in the United States National Museum, Smithsonian Institution, Washington DC. Down, two chromotypes of the species from "Quebrada Corcovada", Mérida, near the type locality. 


\section{REPTILIA}

Orden Squamata

Suborden Amphiasbaenia

FAmilia Amphisbaenidae

Amphisbaena alba (Linnaeus, 1758). Lámina 5A

Material: MZUC 8562, 3 agosto 1964, Cumaná, Estado

Sucre. MZUC 11630, 14 agosto 1963, Cumanacoa, Estado

Sucre.

\section{Suborden Sauria}

\section{Familia Corytophanidae}

Basiliscus basiliscus (Linnaeus, 1758). Lámina 5B

Material: MZUC 11120 (macho adulto), MZUC 11023, 39589 (hembras subadultas), MZUC 11069 (juv), 29 marzo 1964, Río Palmar, Estado Zulia. MZUC 11022 (macho adulto), MZUC 39590, 40165 (hembras adultas), MZUC 39590, 11127 (juv), 4 diciembre 1964, Río en San Cristóbal, Estado Táchira. MZUC 11432 (juv), 26 mayo 1964, El Tukuko, Estado Zulia.

Comentarios: Donoso Barros (1968) considera la subespecie nominal para los ejemplares del estado Zulia (Río Palmar y El Tokuko), mientras que asigna a la subespecie $B . b$. barbouri los de la Cordillera de Mérida (Táchira). Nosotros no consideramos que las diferencias que aduce Ruthven (1914) para separar la especie son suficientes, y hasta que no se realice un estudio comprehensivo seguimos usando el apelativo especifico.

\section{Familia Dactyloidae}

Anolis auratus Daudin, 1802. Lámina 5C

Material: MZUC 11017 (hembra subadulta), 3 mayo 1951, Espino, Estado Guárico (Leg. Kleissen).

Anolis gaigei Ruthven, 1916. Lámina 5D

Material: MZUC 11197, 39348 (juvs), 20 abril 1964, y MZUC 40157 (juv), 26 abril 1964, Sierra de Perijá, Estado Zulia.

Comentarios: La localidad de Perijá es extremadamente vaga, ya que se trata de una cordillera entera. Conocido hasta recientemente en Venezuela como Anolis tropidogaster (Rivas et al. 2012), ha sido ubicado en A. gaigei por Köhler et al. (2012). Los ejemplares están muy deshidratados.

Anolis onca (O'Shaughnessy 1875). Lámina 5E

Material: MZUC 11204 (macho subadulto), 16 junio 1963, Cumanagoto, Estado Sucre. MZUC 11203 (hembra subadulta), 21 agosto 1963, norte de la Isla de Coche, Estado Nueva Esparta. MZUC 11205, 39346 (machos subadultos), 28 enero 1963, Playa Cumanagoto, Estado Sucre.

Anolis planiceps Troschel, 1848. Lámina 5F

Material: MZUC 11201, 39349 (juv), 4-diciembre 1963, Altamira, Estado Barinas. MZUC 11215 (hembra adulta),
5 diciembre 1963, Altamira, Estado Barinas. MZUC 38036, 38 (machos adultos), 38037, 40 (hembras adultas), 29 mayo 1964, Macuro, Estado Sucre. MZUC 38041 (hembra adulta), 38042 (juv), 10 noviembre 1963, Macuro, Estado Sucre. MZUC 38043 (hembra adulta), 38039 (juv), 29 mayo 1964, Macuro, Estado Sucre.

Comentarios: Este taxón ha cambiado de nombre varias veces en los últimos tiempos. Ingresados en el catálogo como Norops nitens o Anolis chrysolepis. Actualmente las poblaciones venezolanas asignadas a nitens o chrysolepis son consideradas Anolis planiceps (D'Angiolella et al. 2011).

\section{Familia Gymnophtalmidae}

Anadia bitaeniata Boulenger, 1903. Lámina 6A

Material: MZUC 11252, 18975 (juvs), 8 diciembre 1963. MZUC 18974 (macho adulto), 18971, 18973 (hembras adultas), 11172, 18970, 18972 (juvs) 23 Junio 1964, todos colectados en el río Mucujún (ver Comentario), Sierra de la Culata (probablemente sobre los $3000 \mathrm{~m}$ ), Estado Mérida.

Comentario: La localidad que aparece en Donoso Barros (1968) para esta especie es Mucují, mientras que en el catálogo de MZUC aparece como Mucujui. La verdad es que no conocemos ningún topónimo como éstos en los páramos merideños. Pero la especie es bien conocida de la naciente del río Mucujún en la Sierra de la Culata, que es fácilmente accesible en vehículo y lo era también en 1963 y 1964, por lo que corregimos la localidad.

Bachia heteropa marcelae Donoso Barros et Garrido, 1964 (= B. heteropa Wiegmann, 1856). Figura 5

Material: MZUC 8578, holotipo macho (6312061 en la publicación original). MZUC 12042 y 12043, paratipos hembras (números respectivos aparecidos en la publicación original 6312062 y 63), colectadas el 12 Diciembre 1963, en La Luz, Estado Barinas.

Comentario: Después de la publicación original (Donoso Barros y Garrido, 1964), Donoso Barros (1968) considera a Bachia marcelae como subespecie de B. lineata, con base a mínimas diferencias en escamación. Dixon (1973), en su revisión comprehensiva del género, considera a Bachia lineata subespecie de $B$. heteropa, así como a marcelae. Bachia heteropa muestra una reducción clinal de este a oeste de la escamación cefálica y del tamaño y número de dedos de las extremidades posteriores. No sería de extrañar que nos halláramos ante un complejo de especies y que marcelae fuera un nombre aplicable a una especie plena (Rivas et al. 2005).

Euspondylus acutirostris (Peters, 1862)

Material: MZUC 11275 (macho adulto), 25 noviembre 1963, Rancho Grande, Parque nacional Henri Pittier, Estado Aragua (G. Medina Leg.). 
Gymnophtalmus sp. aff. speciosus (Hallowell, 1861)

Material: MZUC 8556, 22 septiembre 1963, km 22 a Cumanacoa, estado Sucre. MZUC 8582, 19054-56 (machos adultos), MZUC 19052-53 (hembras adultas), 6 Diciembre 1963. La Luz, Estado Barinas.

Comentario: la especie Gymnophtalmus speciosus es centroamericana. Las poblaciones que existen en todo el norte de Venezuela deben revisarse y muy probablemente correspondan a una o varias especies similares.

Ptychoglossus kugleri Roux, 1927

Material: MZUC 11249 (hembra adulta), noviembre 1964, Rancho Grande, Parque nacional Henri Pittier, Estado Aragua.

Riama achlyens (Uzzell, 1958). Lámina 6B

Material: MZUC 19066 (macho adulto), MZUC 19068 (hembra adulta), MZUC 19070 (macho subadulto), MZUC 19069 (hembra subadulta), MZUC 19067, 19071-72 (juveniles), 14 de febrero de 1964, Rancho Grande, Parque nacional Henri Pittier, Estado Aragua.

Comentarios: Los especímenes colectados por RDB en Rancho Grande fueron identificados como Proctoporus achlyens. No es hasta mucho tiempo después que esta especie se asigna a Riama Gray, 1858 (Doan y Castoe 2005).

Tretioscincus bifasciatus Shreve, 1947. Lámina 6C

Material: MZUC 18081 (macho adulto) 22 de septiembre de 1963, Cumanacoa, Estado Sucre. El resto de especímenes son juveniles sin sexar. MZUC 8551, 8553, 8555, 1907379; 22 septiembre 1963, $22 \mathrm{~km}$ camino a Cumanacoa, Estado Sucre. MZUC 11318, 22 septiembre 1963, Ipures, Estado Sucre.

Comentario: Catalogados bajo la subespecie T. b. kugleri por RDB. Esta subespecie ha sido reportada para las poblaciones venezolanas de la especie, pero ya Marcuzzi (1950) dudaba sobre la validez de la subespecie dada la variabilidad cromotípica de la especie. La lista de reptiles más reciente de Venezuela (Rivas et al. 2012) no considera la subespecie como válida. No obstante, se hace necesaria una revisión del género que podría arrojar resultados inesperados.

Familia Iguanidae

Iguana iguana Linnaeus, 1758. Lámina 6D

MZUC 1114014 de febrero 1963 Cumaná, Estado de Sucre y MZUC 1114126 de agosto de 1963, Cumanacoa, Estado Sucre.

\section{Familia Polychrotidae}

Polychrus aff. marmoratus Cuvier, 1817. Lámina 6E Material: MZUC 11016 (macho), 25 junio 1952, Caracas, Distrito Federal (Leg. J. Roze). MZUC 11251 (macho), 11433, 38033 (hembras), 30 mayo 1964, Macuro, Estado Sucre.

Comentario: Gabriel Ugueto (com. pers.) considera que las poblaciones orientales asignadas a $P$. marmoratus pertenecen a un taxón aun indescrito.

\section{Familia Teitdae}

Comentario: Donoso Barros (1968) reporta varias subespecies de Ameiva ameiva en Venezuela. Esta clasificación también adoptada en el Neotropical Squamata (Peters y Donoso Barros 1970) ha sido ampliamente seguida hasta la reciente revisión de Ameiva en Venezuela por Ugueto y Harvey (2011) en la cual el panorama sistemático cambia sustancialmente. Aquí seguimos la clasificación de estos autores, refiriendo directamente los especímenes a su ubicación sistemática en este momento aceptada.

Ameiva ameiva (Linnaeus, 1758). Lámina 6F

Material: MZUC 11407, 18992 (machos adultos), 18993 (macho subadulto), 18991 (hembra juvenil). Noviembre 1964, Araguaimujo, Orinoco, Estado Delta Amacuro. 


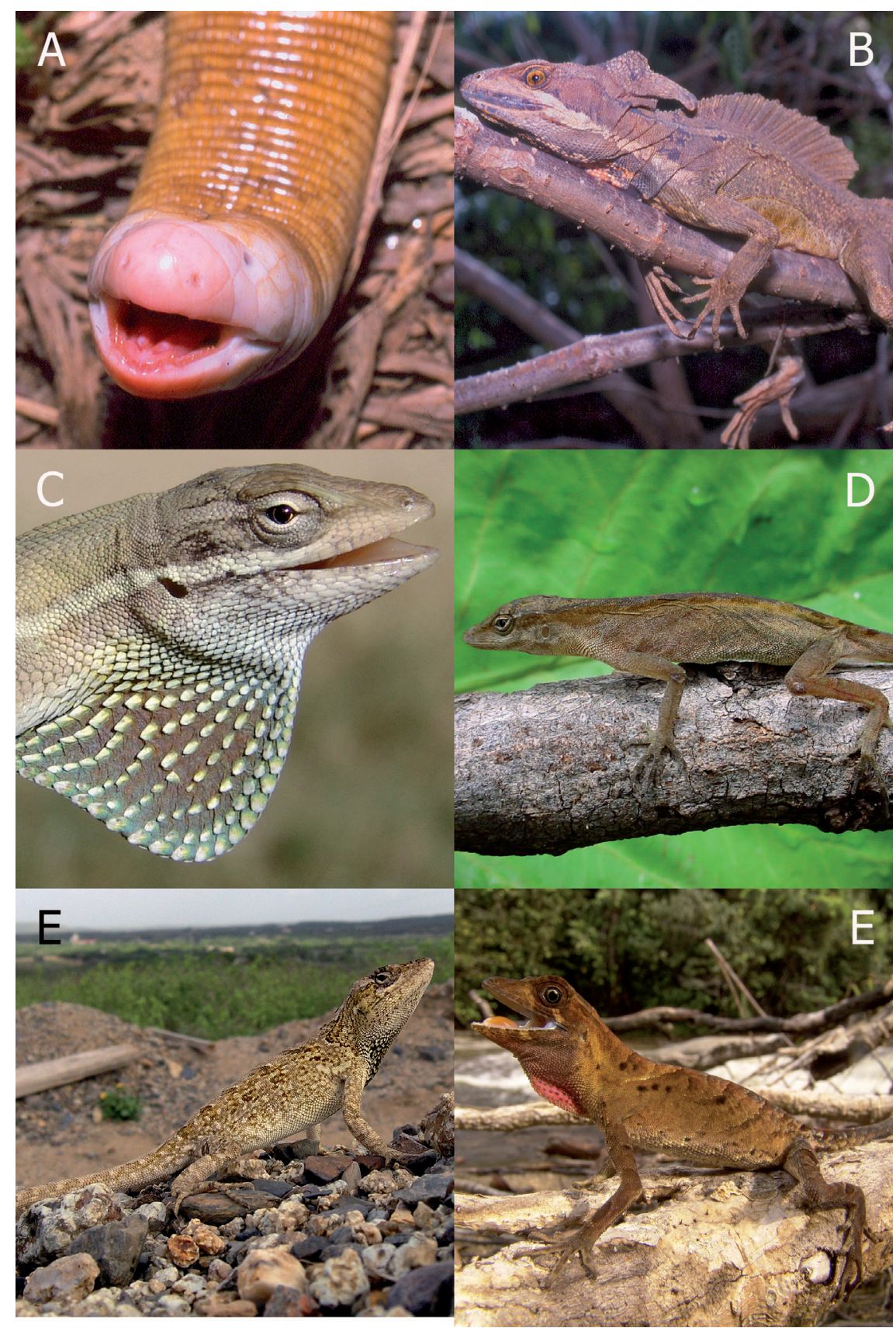

LÁmina 5. A: Amphisbaena alba. Una culebra morrona o de dos cabezas común en el país; llega a alcanzar casi un metro de largo. Son subterráneas pero aparecen después de fuertes lluvias. Limón, Estado Aragua. B: Basiliscus basiliscus. Un lagarto grande, semiacuático que posee la habilidad de correr sobre el agua. Santa María de Caparo, Estado Mérida. C: Anolis auratus. Común en áreas abiertas; el nombre también esconde un complejo taxonómico; San Vicente, Estado Apure. D: Anolis gaigei. Especie poco conocida, sólo común en determinadas zonas de la Hoya del Lago de Maracaibo. Santa Cruz de Mora, Estado Mérida. E: Anolis onca. Especie abundante en zonas xerófitas del norte del país; Península de Paraguaná, Estado Falcón. Foto: Aurelien Miralles. F: Anolis planiceps. Una especie forestal bien repartida por todo el país. La Laja, Sierra de Lema, Estado Bolívar.

Plate 5. A: Amphisbaena alba. A so-called two headed snake, quite common in Venezuela; it reaches almost a meter of length; they are subterranean but usually appear on the Surface after strong rains. Limón, Estado Aragua. B: Basiliscus basiliscus. A large, semi-aquatic lizard, known by its ability to run over the water; Santa María de Caparo, Estado Mérida. C: Anolis auratus, common in open áreas ora 11 the country; this name also hides a taxonomic complex to be solved. San Vicente, Estado Apure. D: Anolis gaigei. Litle known species, only common at determined áreas around the Maracaibo Lake Basin; Santa Cruz de Mora, Estado Mérida. E: Anolis onca, abundant species on xerophytous areas or northern Venezuela; Península de Paraguaná, Estado Falcón. Foto: Aurelien Miralles. F: Anolis planiceps, a welldistributed along the country forest species; La Laja, Sierra de Lema, Estado Bolívar. 


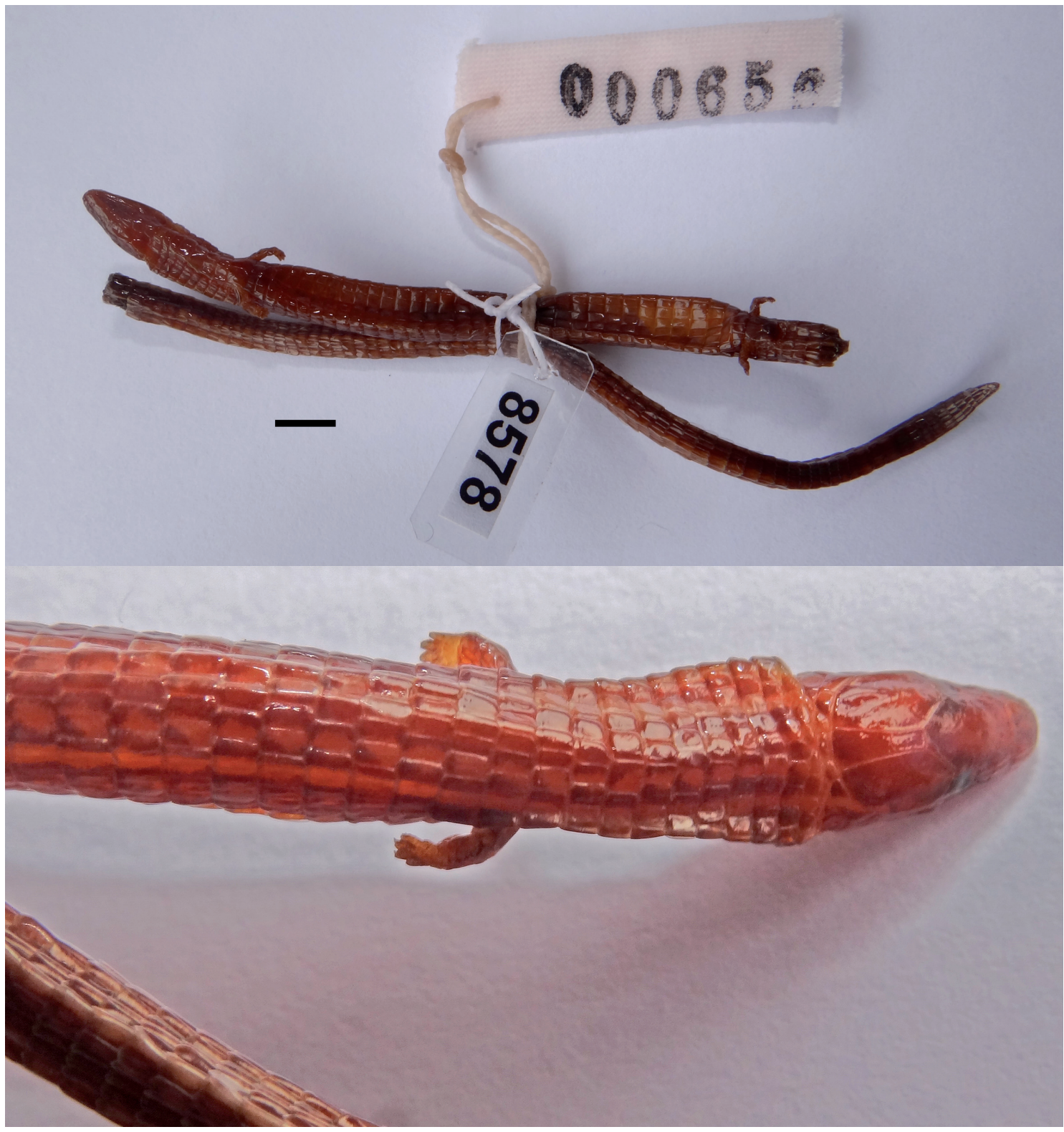

Figura 5. Bachia heteropa marcelae. Holotipo (MZUC 8578). Arriba vista ventral del ejemplar completo. Abajo detalle de la cabeza y parte anterior del cuerpo.

Figure 5. Bachia heteropa marcelae. Holotype (MZUC 8578). Up, ventral view of the whole specimen. Down, head and front of the body details. 
Gayana 79(1), 2015

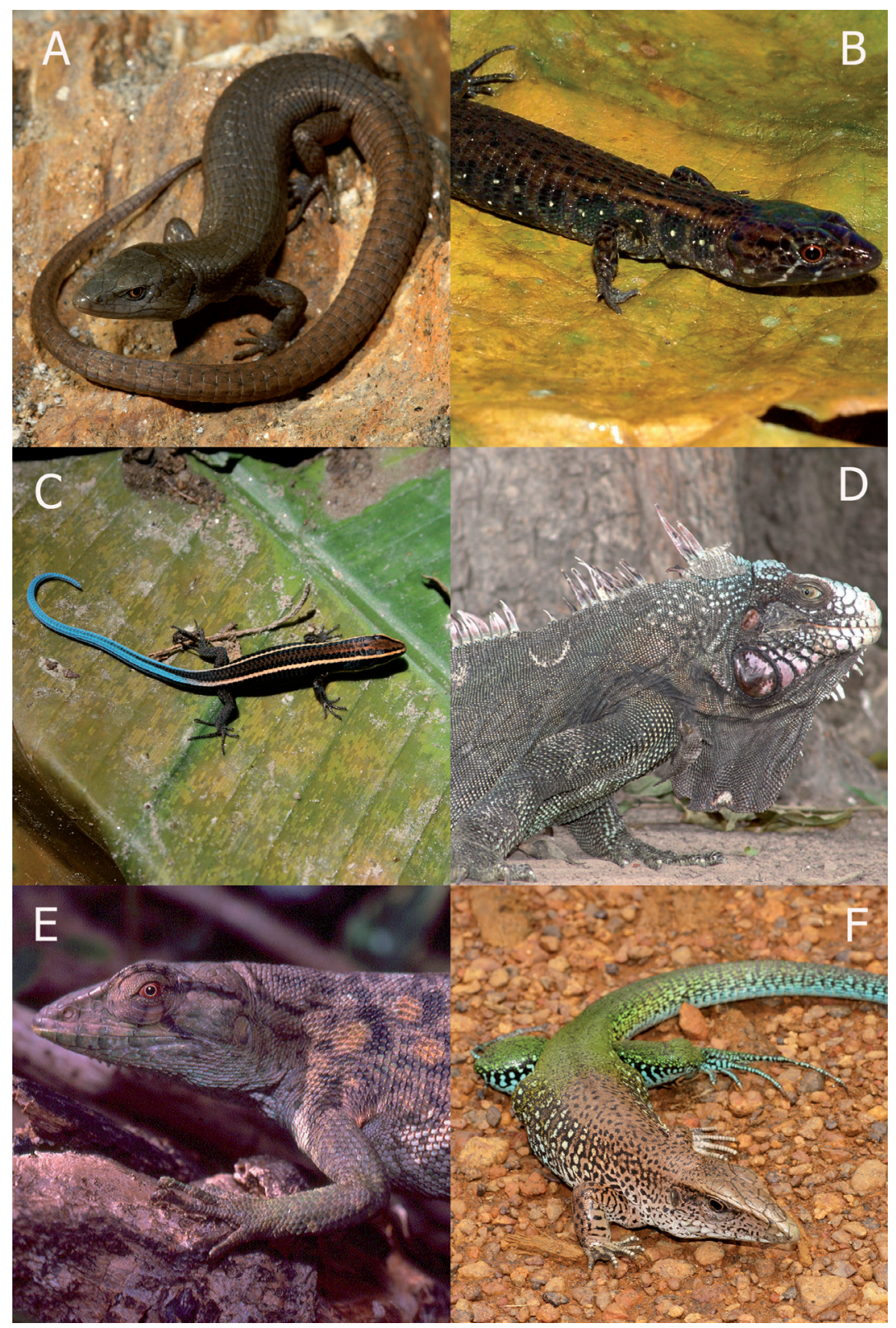

LÁmina 6. A: Anadia bitaeniata. Especie de microteido común en selva nublada y páramos entre 2200 y 3300 m de altitud en los Andes; Páramo de la Culata, Estado Mérida. B: Riama achlyens. Esta pequeña lagartija habita la hojarasca del bosque nublado costero. Rancho Grande, Estado Aragua. Foto: Francisco López. C: Tretioscincus bifasciatus. Una lagartija que era extremadamente común en todo el Norte y NO del país, pero que ha ido enrareciéndose hasta el punto de haber desaparecido de muchos de sus lugares clásicos. San Carlos, Estado Cojedes. Foto: Guillem Chacón. D: Iguana iguana. Especie aún abundante en toda su área de distribución excepto donde la buscan como alimento. Los machos adultos suelen presentar diversas heridas de luchas reproductivas. Hato Cedral, Estado Apure. E: Polychrus marmoratus. Una especie de lagartija arbórea que pocas veces desciende al suelo. Las poblaciones de la península de Paria, como este ejemplar mostrado aquí de Macuro, Estado Sucre, parece que representan una especie por describir. F: Ameiva ameiva. Lagarto de tamaño mediano abundante al sur del Orinoco, donde es frecuente en los claros antropógenos. El Palmar, Imataca, Estado Bolívar.

Plate 6. A: Anadia bitaeniata, Common microteid species at the cloud forest and paramos between 2200 and 3300 masl; Páramo de la Culata, Estado Mérida. B: Riama achlyens, this small lizard inhabits the leaf litter of the coastal cloud forest; Rancho Grande, Estado Aragua. Photo: Francisco López. C: Tretioscincus bifasciatus, a former extremely common lizard throughout all northern Venezuela, it has become much rare nowadays, disapearing from many classic sites; San Carlos, Estado Cojedes. Foto: Guillem Chacón. D: Iguana iguana, an still abundant species through almost all its Distribution area, except where they are searched as food. Adult males can show severe wounds produced during reproductive fights. Hato Cedral, Estado Apure. E: Polychrus marmoratus, an arboreal lizards that rarely descends to the ground; the populations from the Peninsula de Paria, Sucre state, like this one of Macuro seems to represent a different species to describe. F: Ameiva ameiva. A médium sized lizard common south of the Orinoco, where it is frequent on open áreas. El Palmar, Imataca, Estado Bolívar. 
Ameiva atrigularis Garman, 1887. Lámina 7A

Material: MZUC 18989 (macho adulto), 1 setiembre 1963, La Rinconada, Cumanacoa, Estado Sucre. MZUC 11394, 11400 (machos adultos), 11430, 11391 (machos subadultos), 18985 (hembra subadulta), 8 noviembre 1963, Santa Isabel, Estado Sucre. MZUC 11399, (hembras subadultas) 8 febrero 1963, Río Medio, Estado Sucre. MZUC 11130 (juv), 12 octubre 1963, Rio Bohordel, Estado Sucre. MZUC 11119 (juv), 1 Mayo 1963, Hacienda la Rinconada, Cumanacoa, Estado Sucre. MZUC 18984 (juv), 8 noviembre 1963, Santa Isabel, Estado Sucre.

Ameiva bifrontata (Cope, 1862)

Material: MZUC 11112, 11401-02, 19004-05 (machos adultos), 19000-02 (hembras adultas), 19003 (hembra subadulta), 18 Agosto 1963. San Juan Macarapana, Estado Sucre.

Ameiva praesignis (Baird et Girard, 1852). Lámina 7B Material: MZUC 11121 (macho adulto), 11417 y 11431 (machos subadultos), 11396, 18999 (hembras subadultas). 30 octubre 1963, Barinas (orillario), Estado Barinas. MZUC 18997-98 (hembras subadultas), 4 septiembre 1963, El Limón, Estado Aragua.

Cnemidophorus lemniscatus (Linnaeus, 1978). Lámina 7C Material: MZUC 11018, 18953 (machos adultos), 26 julio 1954, Paraguaná, Estado Falcón (Leg. J. Roze). MZUC 11421 (macho adulto), 28 noviembre 1963. San Carlos, Estado Cojedes. MZUC 18954, 18960-62, 18964-65, 18968 (machos adultos), MZUC 11070, 11414, 18955, 18958-59 (hembras adultas), MZUC 11132, 18956-57, 18963, 18969 (juveniles), 9 noviembre 1963, Isla de Patos, Estado Sucre. MZUC 11217 (juv), 2 junio 1968, Cumaná, Estado Sucre. MZUC 1121819 junio 1963, Cumaná, Estado Sucre.

Comentarios: El complejo lemniscatus está lejos de ser completamente bien entendido. Es probable que los ejemplares de Paraguaná correspondan a C. arenivagus.
Cnemidophorus nigricolor Peters, 1873

Material: MZUC 11158, 11058-64. Julio 1963. Los Roques. Comentarios: La serie de esta especie se encuentra muy precariamente conservada, y es difícil aducir el sexo de los ejemplares. Los poros femorales son pocos o nada visibles debido a artefactos de preservación. Los Roques es un archipiélago compuesto por decenas de islas. Lamentablemente no se indica donde fueron colectados estos especímenes. Probablemente en Roque Grande que es donde llega la mayoría de visitantes.

Tupinambis teguixin (Linnaeus, 1758). Lámina 7D Material: MZUC 11423 (hembra adulta), noviembre 1963, Camino a Costa Carúpano, Estado Sucre. MZUC 11624 (hembra adulta), septiembre 1963, carretera CarúpanoCumaná. MZUC 11625 (hembra adulta), 30 octubre 1963, Barinas, Estado Barinas.

\section{Familia Tropiduridae}

Tropidurus hispidus (Spix, 1825). Lámina 7E

Material: MZUC 11020 (juv), 10 octubre 1948, Paraguaná, Estado Falcón (Leg. J. Roze). MZUC 11115 (macho subadulto), 16 junio 1963, Playa Cumaná, Estado Sucre. MZUC 11116 (macho adulto), 18 agosto 1963, San José de Macarapana, cerca de Carúpano, Estado Sucre. MZUC 39294-95 (machos adultos), MZUC 11138 (hembra), MZUC 39297, 39299 (machos jóvenes) y MZUC 39296, 39298, 39300 (juveniles), 14 febrero 1963, Cumaná, Estado Sucre. MZUC 11139, 40159 (juv), 12 diciembre 1963, Golfo de Cariaco, Estado Sucre. MZUC 11434 (hembra) y MZUC 39301 (macho adulto), 14 abril 1963, Ipures, Estado Sucre.

\author{
Suborden Serpentes \\ Familia Boidae \\ Boa constrictor (Linnaeus, 1758). Lámina 7F \\ Material: MZUC 11397, 3 abril 1963, Cerro Colorado, \\ Cumaná, Estado Sucre.
}




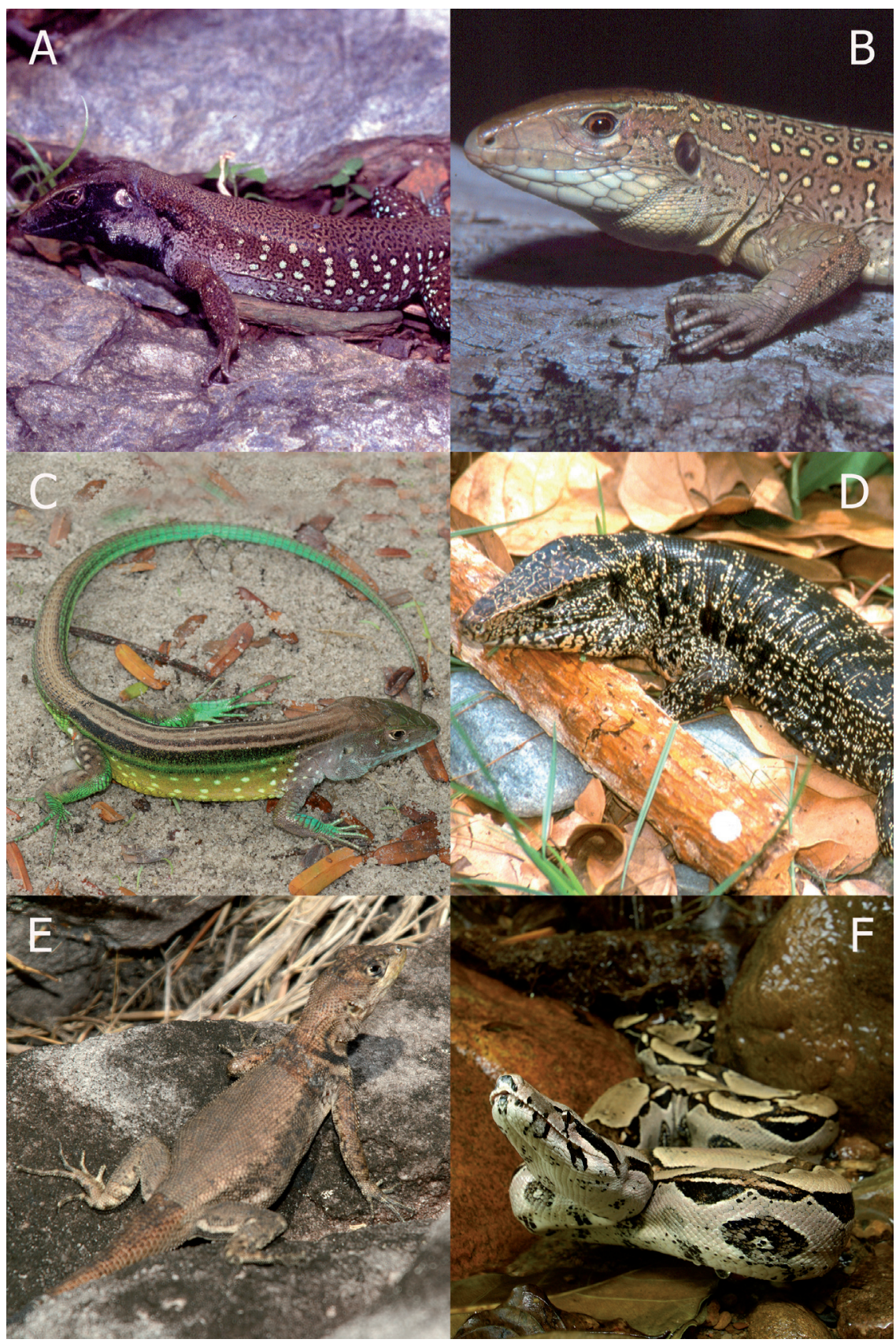

LÁmina 7. A: Ameiva atrigularis. Especie de lagarto de tamaño mediano, que gusta de las zonas antropógenas. Se distribuye por todo el norte y NE del país. Macuro, Estado Sucre. B: Ameiva praesignis. Lagarto mediano que habita zonas de sabana en el centro y NO del país. Hato San Leonardo, Estado Apure. C: Cnemidophorus lemniscatus. Lagartija ampliamente distribuida por el país y de gran complejidad taxonómica. Salto Kawy, Gran Sabana, Estado Bolívar. D: Tupinambis teguixin. Gran lagarto común por todo el territorio nacional, especialmente zonas abiertas. Puerto Concha, Estado Zulia. E: Tropidurus hispidus. Lagarto muy común en zonas secas y abiertas; también antropófilo. Coro, Estado Falcón. F: Boa constrictor. Serpiente constrictora de gran tamaño, alcanzando los 4 m. Comun en casi todas las zonas bajas del país; La Acequia, Estado Barinas.

Plate 7. A: Ameiva atrigularis. Medium sized lizard attracted by anthropogenic áreas, it is distributed along northern Venezuela. Macuro, Estado Sucre. B: Ameiva praesignis. Medium sized lizard living at open áreas like the savannas of los Llanos in the center and NW Venezuela; Hato San Leonardo, Estado Apure. C: Cnemidophorus lemniscatus. A widely distributed lizard, also deserving taxonomic attention. Salto Kawy, Gran Sabana, Estado Bolívar. D: Tupinambis teguixin. Large lizard common around all Venezuela, especially in open áreas. Puerto Concha, Estado Zulia. E: Tropidurus hispidus. A very common lizard of xeric and open áreas, also quite anthropophilous. Coro, Estado Falcón. F: Boa constrictor. Large constrictor snake, reaching 4 m; common in lowlands around the country. La Acequia, Estado Barinas. 
Epicrates maurus (Gray, 1849). Lámina 8A

Material: MZUC 11037, 5 agosto 1963, Cumanacoa, Estado Sucre. MZUC 11087, 41223-24, 20 julio 1963, Cumanacoa, Estado Sucre. MZUC 11393, 11 diciembre 1963, Estado Táchira.

Comentario: Anteriormente considerada una subespecie de Epicrates cenchria. (Passos y Fernandes 2008). BarrioAmorós y Díaz de pascual (2008) reportan Epicrates cenchria y E. maurus simpátricas en el estado Táchira.

\section{Familia Colubridae}

Coluber mentovarius (Dumeril, Bibron et Dumeril, 1854). Lámina 8B

Material: MZUC 11322, diciembre 1963, Barinas, Estado Barinas. MZUC 11572, 29 setiembre 1963, Barinas, Estado Barinas.

Drymarchon corais (Boie, 1827). Lámina 8C

Material: MZUC 11387, 5 abril, 1963, Cumanacoa, Estado Sucre.

Leptophis caerulodorsus (Oliver, 1942). Lámina 8D Material: MZUC 11081, 6 julio 1963, Güiria, Estado Sucre. MZUC 11330, 5 junio 1963, Cumaná, Estado Sucre. MZUC 11358, noviembre 1964, Limón Estado Aragua.

Comentario: Aunque Rivas et al. (2012) no consideran subespecies de L. ahaetulla, es bien conocido que existen al menos tres taxones en Venezuela fácilmente diagnosticables. N.R. Albuquerque (com. pers) en un trabajo aun no publicado (Albuquerque 2008) anuncia la elevación a especies de todas las subespecies reconocidas para L. ahaetulla. Murphy et al. (2013) consideran L. caerulodorsus como especie completa y aquí lo seguimos.

Leptophis occidentalis (Günther, 1859). Lámina 8E Material: MZUC 11316, 10 diciembre, 1963. Táchira (Estado Táchira).

Mastigodryas amarali (Stuart, 1938)

Material: MZUC 11982, 28 julio 19ts63, Cumanacoa, Estado Sucre. MZUC 11086, 6 julio 1963, camino a Güiria, Estado Sucre.

Mastigodryas boddaerti (Sentzen, 1796). Lámina 8F Material: MZUC 11314, 9 febrero 1963, Macuro, Península de Paria, Estado Sucre. MZUC 11374, 41616, Macuro, península de Paria, Estado Sucre. MZUC 11632, 7 noviembre 1963, Isla de Patos, Estado Sucre. MZUC 11315 , 9 febrero 1963, Isla de Patos, Estado Sucre.

Mastigodryas pleei (Dumeril et Bibron, 1854). Lámina 9A Material: MZUC 11029, 14 agosto 1963, Cumaná a Cumanacoa, Estado Sucre. MZUC 11030, 9 marzo 1963, Cerro Colorado, Cumaná, Estado Sucre. MZUC 11066, 9 agosto 1963, Cerro Colorado, Cumaná, Estado Sucre. MZUC 11091, 17 julio 1963, Cerro Colorado, Cumaná, Estado Sucre. MZUC 11102, 5 agosto, 1963, Cumanacoa, Estado Sucre. MZUC 11360, enero 1963, Cumaná, Estado Sucre. MZUC 41623, 28 abril 1963, Cerro Colorado, Cumaná, Estado Sucre. MZUC 11031 (juv), Arica, costa Macuro, Estado Sucre. MZUC 11361, 27 diciembre 1963, Isla Caribe, Estado Sucre. MZUC 11362, 29 de mayo 1963, Camino a Guiría, Estado Sucre.

Oxybelis aeneus (Wagler, 1824). Lámina 9B

Material: MZUC 11060, 7 junio 1963, camino de Guiria a Rio caribe, Estado Sucre. MZUC 11064, 28 de mayo, 1964, Macuro, Estado Sucre: MZUC 11089, Barinas, Estado Barinas. MZUC 11090, 6 julio 1963, Rio Caribe, Estado Sucre. MZUC 11378, 10 de septiembre, 1963. Caripito, Estado Monagas.

Oxybelis fulgidus (Daudin, 1803). Lámina 9C Material: MZUC 11059, 7 febrero 1963; y MZUC 11079, 10 de noviembre 1963, Isla de Patos, Estado Sucre.

Spilotes pullatus (Linnaeus, 1758). Lámina 9D

Material: MZUC 11092, 5 junio 1963, Cumaná, Estado Sucre. MZUC 11101, 8 febrero 1963, Carúpano, Estado Sucre. MZUC 11012, 8 agosto 1963, Cumanacoa, Estado Sucre.

Tantilla melanocephala (Linnaeus, 1758). Lámina 9E Material: MZUC 11356, 24 octubre 1963, Guarenas, Estado Miranda.

\section{Familia Dipsadidae}

Erythrolamprus bizona Jan, 1863. Lámina 9F

Material: MZUC 11391, 10 diciembre 1963, Mirador de San Cristóbal, Estado Táchira. 


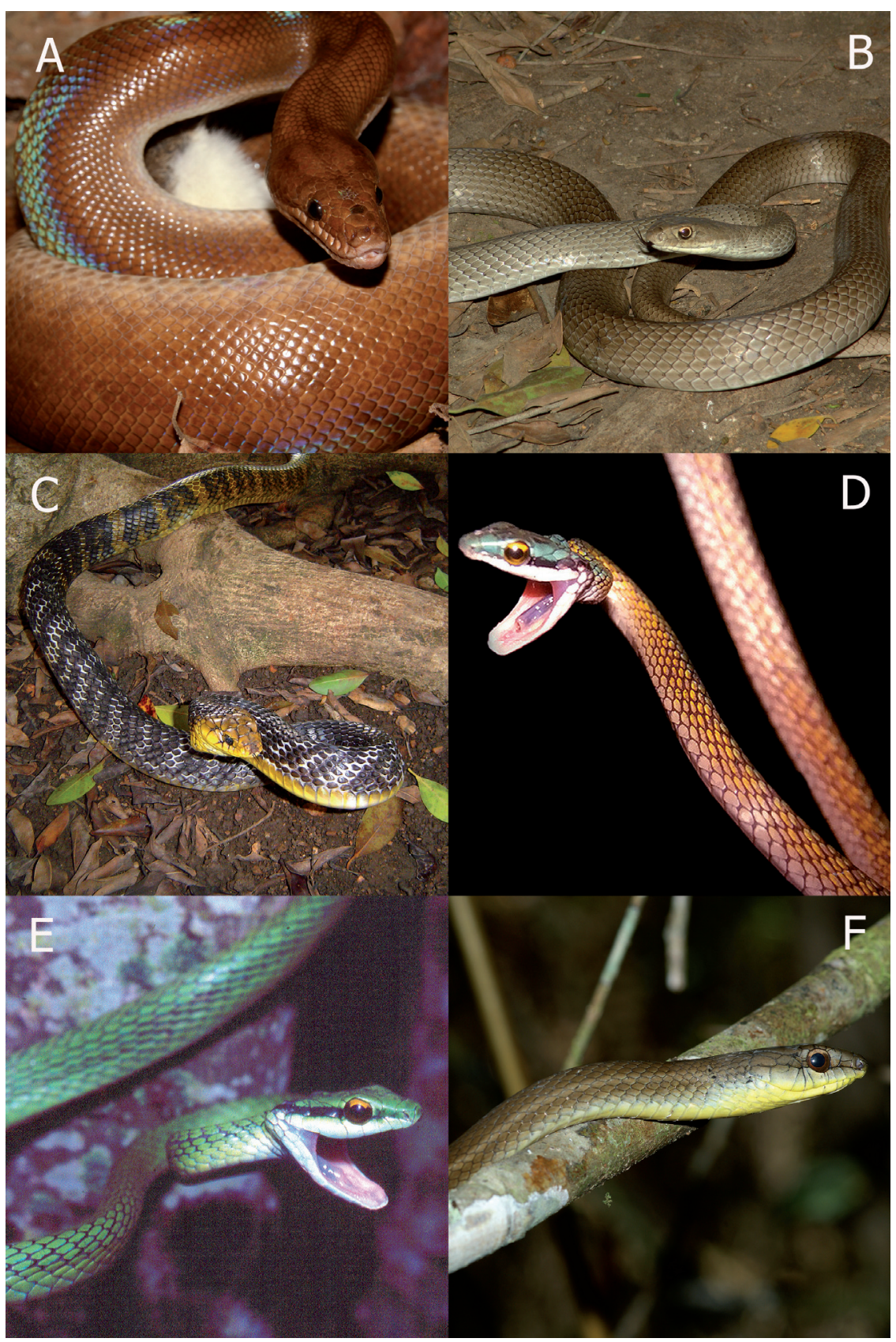

LÁmina 8. A: Epicrates maurus. Una boa terrestre habitante de zonas abiertas. Isla de Margarita, Estado Nueva Esparta. Foto cortesía de Quetzal Dwyer. B: Coluber mentovarius. Culebra de espacios abiertos muy rápida y especialista en mamíferos terrestres. Barquisimeto, Estado Lara. C: Drymarchon corais. Gran culebra que puede alcanzar los $3 \mathrm{~m}$, terrestre, de hábitat abierto pero escasa. D: Leptophis caerulodorsus. Culebra bejuca común en el centro y norte del país. Es opistoglifa y aunque no suele morder, realiza un exhibición defensiva abriendo la boca y amenazando. Triunfo, Estado Bolívar. E: Leptophis occidentalis. Especie similar a la anterior pero de cuerpo completamente verde, y más larga. Habita el NO del país. Estado Zulia. F: Mastigodryas boddaerti. Culebra muy común en todo el país, habitando desde el nivel del mar hasta los 3000 m, en zonas preferentemente boscosas; es bastante antropófila. Calderas, Estado Barinas.

Plate 8. A: Epicrates maurus. A terrestrial médium sized boa, inhabiting open áreas; Isla de Margarita, Estado Nueva Esparta. Photo courtesy of Quetzal Dwyer. B: Coluber mentovarius. Snake typical from open spaces, very quick, feeding on mammals and lizards. Estado Lara. C: Drymarchon corais. Large snake that can reach $3 \mathrm{~m}$; not common, living at open áreas. Represa Caruachi, Estado Bolívar. D: Leptophis caerulodorsus. A common parrot snake in the center and north of the country. It is opistoglyphous and though it usually does not bite, like to make a defensive display opening the mouth. Triunfo, Estado Bolívar. E: Leptophis occidentalis. Similar species but completely Green and longer; it inhabits the NW of the country. El Manzano, Estado Lara. F: Mastigodryas boddaerti. A very common snake in all the country, from sea level to 3000 masl, in forests and dirturbed áreas. Calderas, Estado Barinas. 


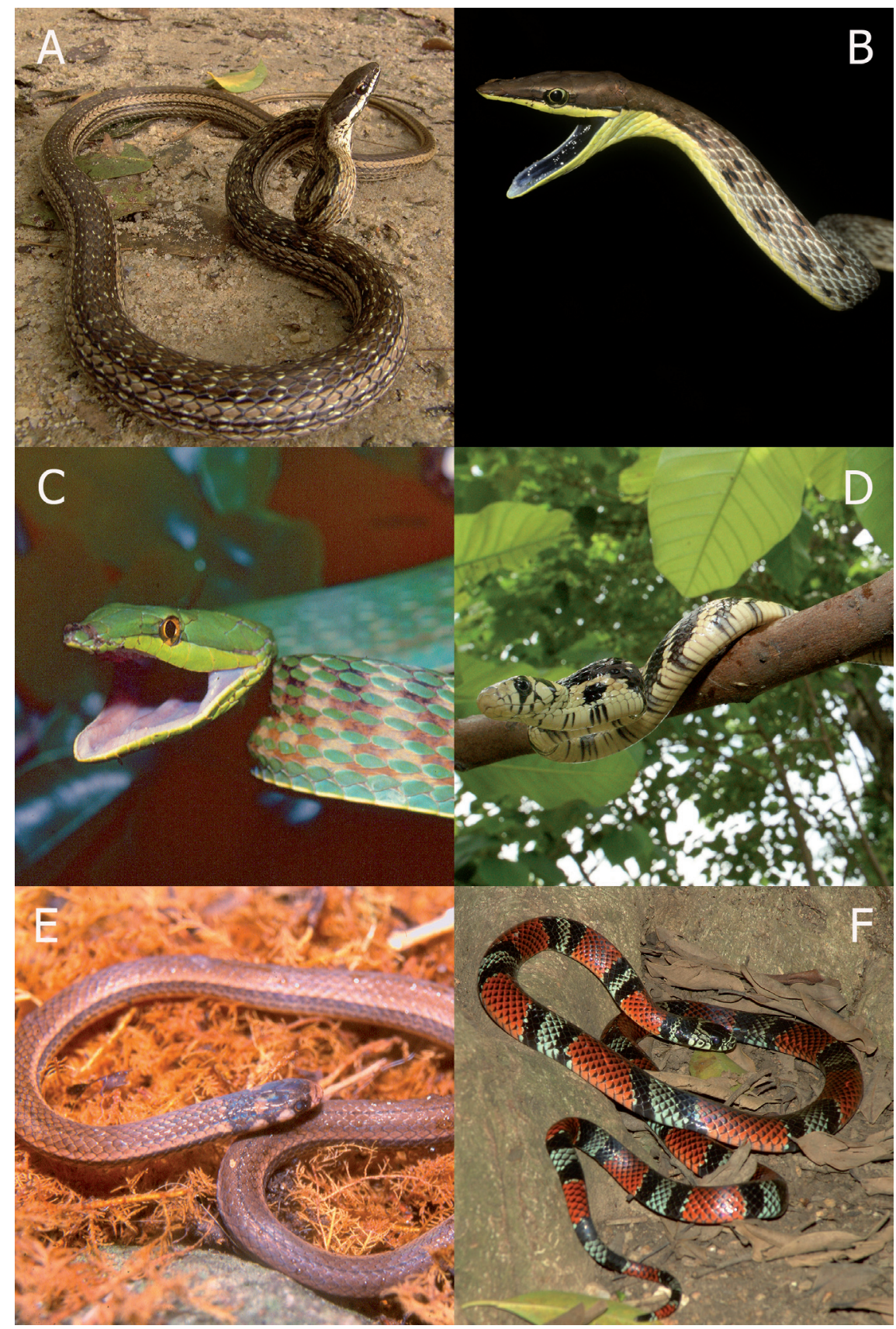

LÁmina 9. A: Mastigodryas pleei. Especie muy común en zonas xerófitas de todo el país. Cuidad Bolívar, Estado Bolívar. B: Oxybelis aeneus. Una de las especies de serpientes de mayor distribución Neotropical, habita tanto zonas xerófitas como selváticas, donde se confunde con una liana. Isla Bastimentos, Panamá. C: Oxybelis fulgidus. Especie relacionada a la anterior, pero de color verde, habita selvas deciduas, secas y siempreverdes. Puerto Ordaz, Estado Bolívar. D: Spilotes pullatus. Gran culebra arbórea, que alcanza los $3 \mathrm{~m}$. Es una cazadora activa de aves, reptiles y mamíferos arborícolas. Maracay, Estado Aragua. E: Tantilla melanocephala. Culebra muy pequeña que no supera los $40 \mathrm{~cm}$; semi fosorial. Caparo, Estado Barinas. F: Erythrolamprus bizona. Una falsa coral relativamente común en el norte del país. Es una especie ofiófaga. Barquisimeto, Estado Lara.

Plate 9. A: Mastigodryas pleei. A common species in xerix áreas of all the country. Cuidad Bolívar, Estado Bolívar. B: Oxybelis aeneus. One of the most widely distributed Neotropical snakes, it inhabits xeric as well as rainforest conditions, where it confounds itself with a vine. Isla Bastimentos, Panamá. C: Oxybelis fulgidus. A related species but completely green, inhabiting only rain or dry forest situations. Puerto Ordaz, Estado Bolívar. D: Spilotes pullatus. Large arboreal snake, reaching $3 \mathrm{~m}$ long; it is an active hunter of birds and arboreal mammals. Maracay, Estado Aragua. E: Tantilla melanocephala. Small snake that reaches $40 \mathrm{~cm}$; semi fossorial. Caparo, Estado Barinas. F: Erythrolamprus bizona. A false coral snake relatively frequent along northern Venezuela; ophiophagous, feeding on other snakes. Sanare, Estado Lara. 
Erythrolamprus melanotus (Shaw, 1802). Lámina 10A

Material: MZUC 11032, 24 octubre 1963, Barlovento, Estado Miranda. MZUC 11099, 2 marzo 1964, Barinas, Estado Barinas. MZUC 11313, 8 diciembre 1063, La Luz, Estado Barinas. MZUC 11320, 22 julio 1963, Cumanacoa, Estado Sucre. MZUC 11321, 2 marzo 1963, La Fría, Estado Táchira.

Comentario: El género Liophis, dentro del cual era asignada esta especie hasta recientemente, es considerado sinónimo de Erythrolamprus por Grazziotin et al. (2012).

Helicops scalaris Jan, 1865. Lámina 10B

Material: MZUC 12340, 25 marzo 1963, Tukuko, Perijá, Estado Zulia.

Comentario: Introducido al catálogo como Helicops polylepis.

Imantodes cenchoa (Linnaeus, 1758). Lámina 10C

Material: MZUC 11024, 10 diciembre 1963, Valera, Estado Trujillo.

Leptodeira annulata (Hallowell, 1845). Lámina 10D Material: MZUC 11368, 4 diciembre 1963, Barinas, Estado Barinas. MZUC 11096, 11098, 11117, 11122, 1 de marzo, 1963, Puerto Nutrias, Estado Barinas. MZUC 11077, 6 diciembre 1963, Puerto Nutrias, Estado Barinas. MZUC 11080, 6 diciembre, 1963, Ciudad de Nutrias, Estado Barinas. MZUC 11084, 20 junio, 1963, Cumanacoa, Estado Sucre. MZUC 11095, 5 agosto, 1963, Cumaná, Estado Sucre. MZUC 11327, 11 mayo 1963, Cumanacoa, Estado Sucre. MZUC 11328, 28 noviembre 1963, San Carlos, Estado Cojedes. MZUC 11376, 24 octubre 1963, Guárico, Estado Guárico. MZUC 11379, 10 diciembre 1963, La Tendida, Estado Táchira. MZUC 11392, 5 abril 1963, Cerro Colorado, Cumaná, Estado Sucre. MZUC 40160, 29 mayo 1964, Macuro, Estado Sucre.

Lygophis lineatus (Linnaeus, 1758). Lámina 10E

Material: MZUC 11094, 8 diciembre 1964, Barlovento, Estado Miranda. MZUC 11355, 3 diciembre 1963, Barinitas, Estado Barinas.

Ninia atrata (Hallowell, 1845). Lámina 10F

Material: MZUC 8799, enero 1964, Caracas, Distrito Federal.

Oxhyropus petolarius (Linnaeus, 1758). Lámina 11A Material: MZUC 8685, 27 de octubre, 1963, Rancho Grande, Estado Aragua.

Comentario: Conocido hasta Savage (2011) como O. petola. Este autor expone la prioridad de petolarius.

Phimophis guianensis (Troschel, 1848). Lámina 11B Material: MZUC 11026, 11366, 12 octubre 1963, Golfo de Cariaco, Estado Sucre. MZUC 11065, 25 junio 1963, Cumaná, Estado Sucre. MZUC 11085, julio 1963, Cerro Colorado, Estado Sucre. MZUC 11324, 8 diciembre 1963, Barinas, Estado Barinas.

Pseudoboa neuwiedi (Dumeril, Bibron et Dumeril, 1854). Lámina 11C

Material: MZUC 11035, 7 octubre 1963, Cumaná, Estado Sucre. MZUC 11036, 10 febrero 1964, Puerto La Cruz, Estado Sucre. MZUC 11097, 2 de marzo, 1963, Puerto Nutrias, Estado Barinas. MZUC 11133, 13 agosto 1963, Cumanacoa, Estado Sucre. MZUC 11325, 8 diciembre 1963, Barinas, Estado Barinas. MZUC 11326, 28 noviembre 1963, San Carlos, Estado Cojedes. MZUC 11364, 10 diciembre 1963, Guayabones, Estado Mérida. MZUC 11373, 8 mar4zo 1963, Cumaná, Estado Sucre.

Sibon nebulata (Linnaeus, 1758). Lámina 11D

Material: MZUC 11025, 7 diciembre 1963, La Mitusús, Estado Mérida. MZUC 11076, noviembre 1963, Rancho Grande, Estado Aragua. MZUC 11381, 6 diciembre, 1963, Mérida, Estado Mérida.

Thamnodynastes dixoni Bailey et Thomas, 2007. Lámina $11 \mathrm{E}$

Material: 11370, 1 diciembre 1963, Barinas, Estado Barinas. MZUC 11382, 7 diciembre 1963, San Lorenzo, La Luz, Estado Barinas.

Comentario: Introducidos al catálogo como T. pallidus. Una reciente revisión del género Thamnodynastes en el norte de Suramérica (Bailey y Thomas 2007) asigna los ejemplares de los Llanos venezolanos a $T$. dixoni.

\section{FAMilia LePTOTYPHLOPIDAE}

Epictia albifrons (Wagler, 1824)

Material: MZUC 8619, 7 octubre 1963; MZUC 8621, 7 junio 1963; MZUC 8622, 12 junio 1963, y MZUC 8536, enero 1962, todos de Cumaná, Estado Sucre.

\section{Familia Elapidae}

Micrurus isozonus (Cope, 1860)

Material: MZUC 11332, 6 noviembre, 1963, Santa Rosa, Estado Barinas.

\footnotetext{
Familia Viperidae

Crotalus durissus cumanensis (Humboldt, 1833). Lámina $11 \mathrm{~F}$

Material: MZUC 8423, junio 1963, Cumaná, Estado Sucre. MZUC 11038, 41622, camino Cumaná a Cumanacoa, Estado Sucre. MZUC 11078, 29 setiembre, 1963, Pertigaleta, Estado Sucre. MZUC 11329, 14 diciembre, 1963, Pan de Azúcar, Estado Guárico. MZUC 11372, 17 setiembre 1963, Cumanacoa, Estado Sucre. MZUC 1138010 diciembre 1963, Golfo Cariaco, Estado Sucre.
} 


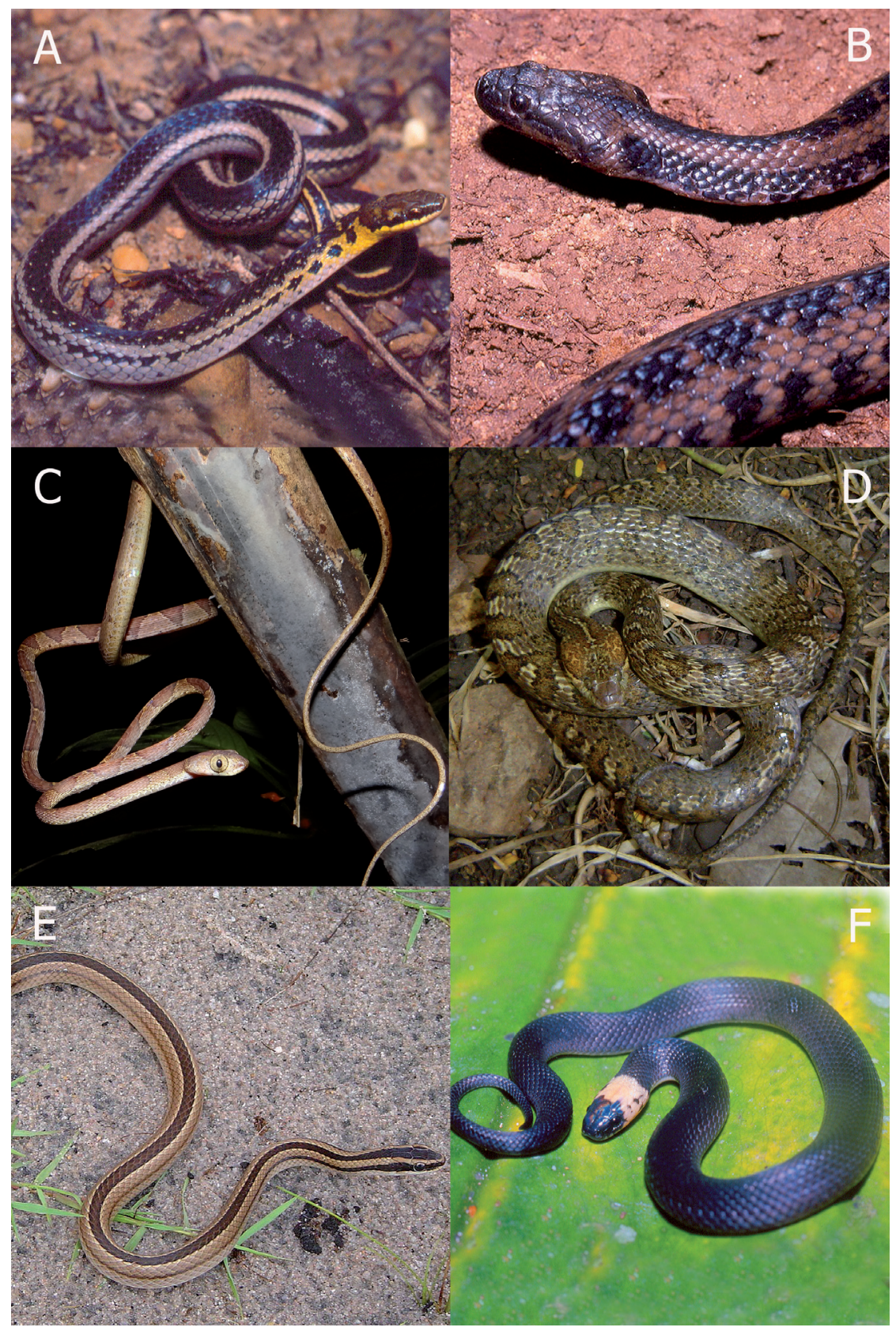

LÁminA 10. A: Erythrolamprus melanotus. Una culebra muy abundante en el centro y norte del país, de zonas semi arboladas y abiertas, San Vicente, Estado Apure. B: Helicops scalaris. Serpiente semi-acuática, común en zonas anegadas de la Hoya del Lago de Maracaibo. El Vigía, Estado Mérida. C: Imantodes cenchoa. Culebra extraordinariamente alargada, nocturna, que se mueve lentamente por el follaje buscando lagartijas dormidas y ranas. Es común en todo el país. Sarapiquí, Costa Rica. D: Leptodeira annulata. Otra de las culebras más comunes en todo el país, habita casi todo tipo de hábitat, desde selvático a xerófito; no obstante, se reconoce que este nombre engloba un complejo de especies. Barquisimeto, Estado Lara. E: Lygophis lineatus. Culebrita común en zonas abiertas, cazadora de ranas. Puerto Ayacucho, Estado Amazonas. F: Ninia atrata. Una de las especies más pequeñas de serpientes del país. Muy común en todo el norte, incluso en ciudades como Caracas. Rancho Grande, Estado Aragua.

Plate 10. A: Erythrolamprus melanotus. A very abundant species in northern and central Venezuela, from open spaces. San Vicente, Estado Apure. B: Helicops scalaris. A semi-aquatic snake, common in swamps of the Maracaibo lake Basin. El Vigía, Estado Mérida. C: Imantodes cenchoa. Snake extraordinarily elongated, that moves slowly at night looking for sleeping lizards and frogs. It is common in all the country. Sarapiquí, Costa Rica. D: Leptodeira annulata. Another of the most common snakes in all the country; it inhabits allmost all type of hábitat, from rainforest to xeric. However, this names hides a taxonomic complex. Barquisimeto, Estado Lara. E: Lygophis lineatus. Small snake common in open areas, where it hunts mainly frogs. Puerto Ayacucho, Estado Amazonas. F: Ninia atrata. One of the smaller snakes in the country; quite common along the north, inclusive in cities like Caracas. Rancho Grande, Estado Aragua. 


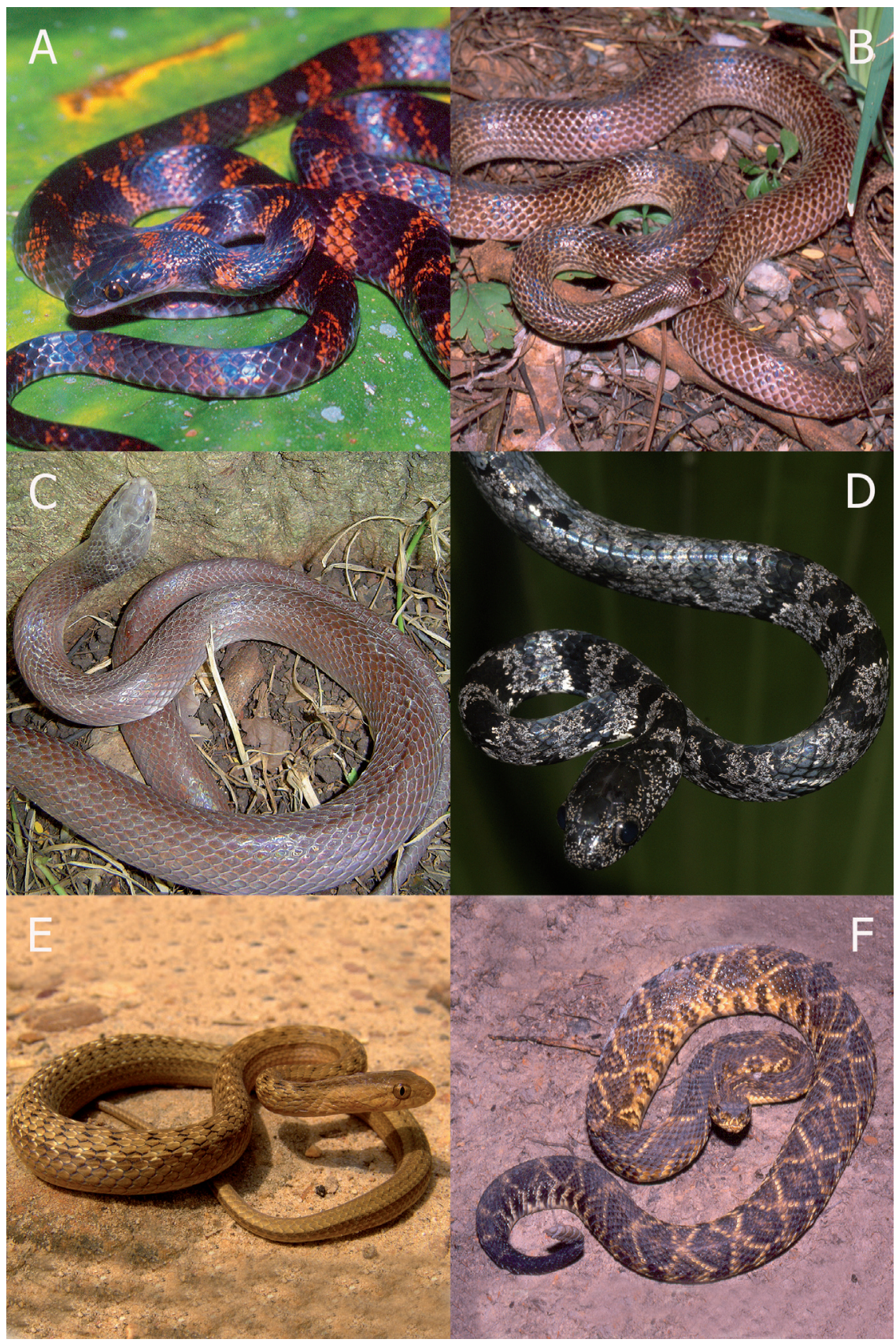

LÁmina 11. A: Oxyrhopus petolarius. Falsa coral muy variable en patrón; inofensiva. Común en todo el país. Rancho Grande, Estado Aragua. B: Phimophis guianensis. Culebra fácilmente reconocible por su hocico apuntado hacia arriba, que le permite excavar el subsuelo. Barquisimeto, Estado Lara. C: Pseudoboa neuwiedi. Culebra opistoglifa pero inofensiva, común en casi todo el país. Su coloración juvenil se confunde con la de Clelia clelia. Barquisimeto, Estado Lara. D: Sibon annulatus. Pequeña culebra caracolera muy común en todo el país, hasta $\operatorname{los} 2500 \mathrm{~m}$. Arenal, Costa Rica. E: Thamnodynastes dixoni. Pequeña culebra opistoglifa y relativamente ponzoñosa. Común en los Llanos venezolanos. San Vicente, Estado Apure. F: Crotalus durissus cumanensis. Una víbora de tamaño mediano a grande, hasta 2 m de longitud. Común en zonas abiertas y xerófitas del país. Muy tóxica. San Vicente, Estado Apure.

Plate 11. A: Oxyrhopus petolarius. A very variable false coral snake, completely harmless. Common in all the country. Rancho Grande, Estado Aragua. B: Phimophis guianensis. Easily recognizable snake, by its pointed snout looking upwards, what permits it to dig in the ground. Barquisimeto, Estado Lara. C: Pseudoboa neuwiedi. Opistogliphous but harmless snake, common in all the country; its juvenile coloration is very similar to that of Clelia clelia. Barquisimeto, Estado Lara. D: Sibon nebulata. Small snail-eater snake, very common in all the country, up to 2500 masl. Arenal, Costa Rica. E: Thamnodynastes dixoni. Small, opistogliphous and agressive snake, relatively venemous. Common in the Venezuelan Llanos. San Vicente, Estado Apure. F: Crotalus durissus cumanensis. A médium to large snake, reaching $2 \mathrm{~m}$ long. Common in some open and xerix áreas. Very toxic and dangerous. San Vicente, Estado Apure. 
Botrops sp. cf asper. Lámina 12A

Material: MZUC 11389, 6 setiembre 1964, Barlovento, Estado Miranda. MZUC 11427, 16 noviembre 1963, Rio Chico, Estado Miranda.

Comentario: El taxón relacionado a $B$. asper habitante del norte de Venezuela ha sido denominado B. colombiensis, más el nombre actualmente no es aplicable (ver Rivas et al. 2012).

\section{Bothrops aff. atrox}

Material: MZUC 11013, 11388, noviembre 1963, La Luz, Estado Barinas.

Comentario: La única especie que habita las selvas de los Llanos altos, donde la localidad de La Luz está situada, es un taxón aún no claramente definido. Relacionado con atrox, ha sido bautizado como $B$. isabelae de manera inapropiada. Pero no existe un holotipo depositado en un museo, y hasta que no se redescriba con material topotípico, este taxón debe denominarse $B$. aff. atrox, ya que genéticamente es a la especie a la que más se asemeja (Fenwik et al. 2009).

Bothrops venezuelensis Sandner Montilla, 1952. Lámina 12B

Material: MZUC 11109, julio 1963, Rancho Grande, Estado Aragua. MZUC 11111, noviembre 1964, Rancho Grande, Estado Aragua. MZUC 11395, 9 febrero 1963, Altamira, Estado Barinas. MZUC 11088, 20 junio, 1963, Altamira, Estado Barinas.

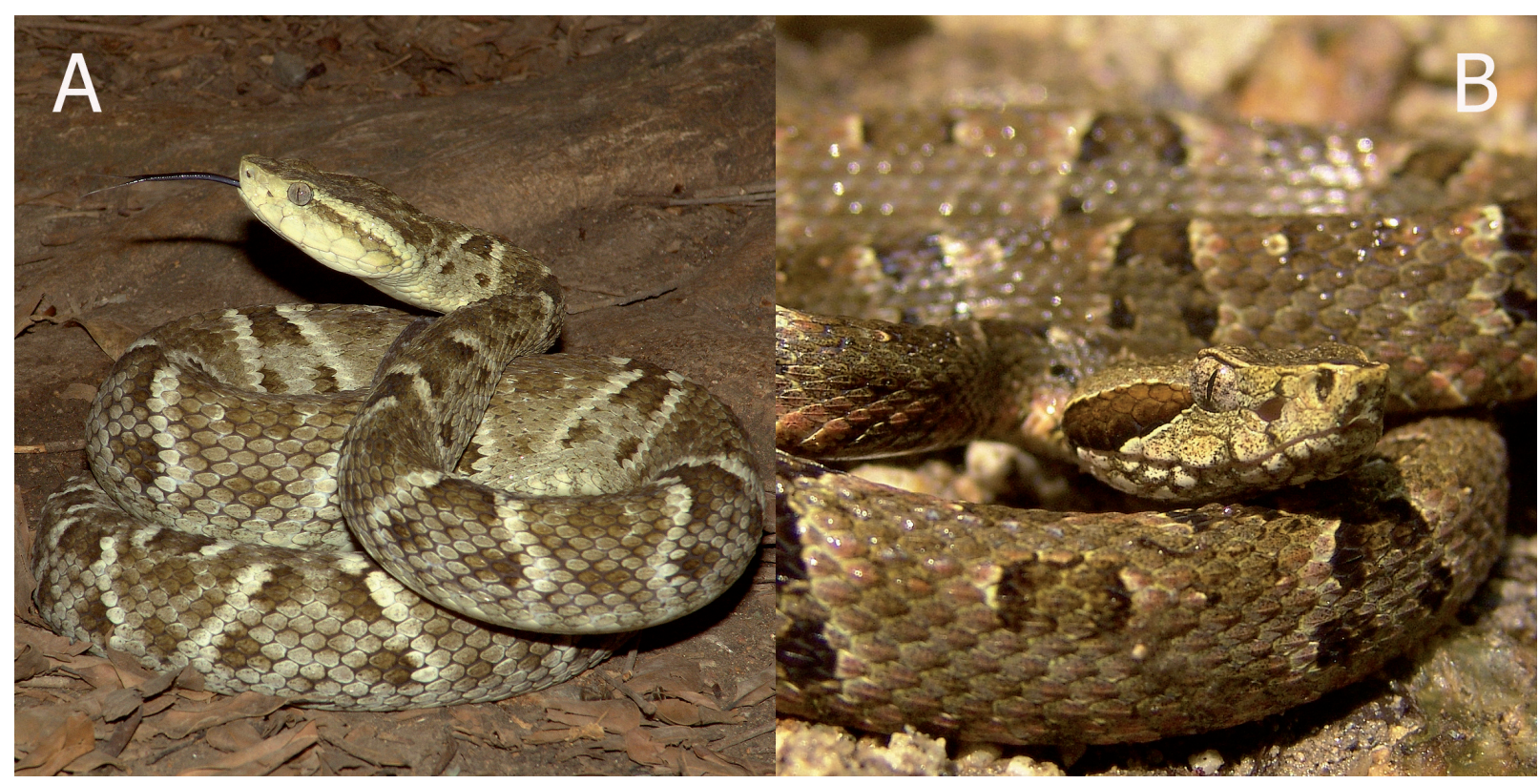

LÁmina 12. A: Bothrops sp. cf. asper. Una de las víboras más comunes en el norte del pais, en zonas bajas y medias de la Cordillera de la Costa, y del piedemonte andino llanero. Extremadamente peligrosa por su mimetismo y abundancia. Barquisimeto, Estado Lara. B: Bothrops venezuelensis. Especie de media montaña, entre selva pluvial y nublada. Bastante común en la Cordillera de la Costa y en el piedemonte llanero de los Andes. Río Frío, Estado Mérida.

Plate 12. A: Bothrops sp. cf. asper. One of the most common vipers through northern Venezuela, in low and uplands of the Coastal chain and the Andean piedmont. Extremely dangerous by its mimetism and abundance. Tarabana, Barquisimeto, Estado Lara. B: Bothrops venezuelensis. This is a species occurrynig ar uplands, between rain and cloud forests; quite common in the Cordillera de la Costa and Andes. Río Frío, Estado Mérida. 


\section{AGRADECIMIENTOS}

Gabriel Ugueto compartió el interés e información sobre ciertos taxones; Diego Alejandro Flores nos asistió con toponimia del Estado Sucre. Las fotos del holotipo de Pristimantis lancinii fueron facilitadas por James Poindexter del Smithsonian USNM. El trabajo de CLBA en la Universidad de Concepción ha sido facilitado por el proyecto DIUC 20611372-3 (JCO).

\section{BIBLIOGRAFÍA}

Adalsteinsson, S.A., W.R. Branch, S. Trape, L.J. Vitt, \& Hedges. S.B. 2009. Molecular phylogeny, classification, and biogeography of snakes of the family Leptotyphlopidae (Reptilia, Squamata). Zootaxa 2244:1-50.

Albuquerque, N.R. 2008. Revisao taxonómica das subespecies de Leptophis ahaetulla (Linnaeus, 1758) (Serpentes, Colubridae). Tesis de Doutorado. Pontificia Universidade Catolica do Rio Grande do Sul.

Bailey, J.R. \& Thomas, R.A. 2006. A revision of the South American snake genus Thamnodynastes Wagler, 1830 (Serpentes: Colubridae, Tachymenini). II. Three new species from northern South America, with further descriptions of Thamnodynastes gambotensis PérezSantos and Moreno and Thamnodynastes ramonriveroi Manzanilla and Sánchez. Memoria de la Fundación La Salle de Ciencias Naturales 166:7-27.

Barrio-Amorós, C.L. 2004a. Atelopus mucubajiensis still survives in the Andes of Venezuela. Preliminary report. FROGLOG 66: 2-3.

Barrio-Amorós, C.L. 2004b. Amphibians of Venezuela, Systematic list, Distribution and References; an Update. Revista Ecología Latino Americana 9(3):1-48.

Barrio-Amorós, C.L. \& Díaz de Pascual, A. 2008. Notes on Geographic Distribution: Reptilia: Boidae: Epicrates cenchria cenchria: Distribution Extension. Checklist 4(3):243-247.

Barrio-Amorós, C.L. \& Kaiser, H. 2008. Distribution of Strabomantis biporcatus (Anura: Terrarana) in northern Venezuela, with comments on its phenotypic variation. Salamandra 44(4):248-254.

Barrio-Amorós, C.L., Rivas, G., Molina, C. \& Kaiser, H. 2006. Mannophryne trinitatis (Anura: Dendrobatidae) is a Trinidadian single-island endemic. Herpetological Review 37(3):298-299.

Barrio-Amorós, C.L., Santos, J.C. \& Molina, C.R. 2010a. An addition to the Diversity of dendrobatid frogs in Venezuela: description of three new collared frogs (Anura: Dendrobatidae: Mannophryne). Phyllomedusa 9(1):3-35.

Barrio-Amorós, C.L., Rivas, G.A., Molina, C., Santos, J.C. \& KAISER, H. 2010b. Intraspecific variation in the Endangered frog Mannophryne riveroi (Anura, Dendrobatidae, Aromobatinae), with comments on coloration and natural history. Herpetological Notes 3:151-160.

Bonnacorso, E., Guayasamin, J.M., Méndez, D. \& Speare, R. 2003. Chytridiomycosis as a possible cause of population declines in Atelopus cruciger (Anura: Bufonidae).
Herpetological Review 34:331-334.

D’Angiolella, A.B., Gamble, T., Avila-Pires, T.C.S., Colli, G.R., Noonan, B.P. \& VitT, L.J. 2011. Anolis chrysolepis Duméril and Bibron, 1837 (Squamata: Iguanidae), revisited: molecular phylogeny and taxonomy of the Anolis chrysolepis species group. Bulletin of the Museum of Comparative Zoology 160:35-63.

DiXon, J.R. 1973. A systematic review of the teiid lizards, genus Bachia, with remarks on Heterodactylus and Anotosaura. University of Kansas, Museum of Natural History. Miscellaneous Publication 57:1-47.

Donn, T.M. \& Castoe, T.A. 2005. Phylogenetic taxonomy of the Cercosaurini (Squamata: Gymnophthalmidae), with new genera for species of Neusticurus and Proctoporus. Zoological Journal of the Linnean Society 143:405-416.

Donoso Barros, R. 1964. A new species of dendrobatid frog, Prostherapis riveroi from Venezuela. Caribbean Journal of Science 4(4):485-489.

Donoso Barros, R. 1966. Hyla robersimoni, nuevo hylidae de Venezuela. Boletín del Museo Nacional de Historia Natural, Chile 29(2):37-43.

Donoso Barros, R. 1968. Un nuevo anuro de los Andes de Venezuela. Caribbean Journal of Science 8 (1-2): 31-34.

Donoso Barros, R. \& Garrido, A. 1964. Nuevo Teiidae de Venezuela, Bachia marcelae, nov. sp. Publicaciones Ocasionales del Museo de Ciencias Naturales, Caracas, Venezuela 8:1-8.

Duellman, W.E. 1977. Liste der rezenten Amphibien und Reptilien. Hylidae, Centrolenidae, Pseudidae. Das Tierreich 95:1225.

Faivovich, J., Haddad, C.F.B., García, P.C.D.A., Frost, D.R., Campbell, J.A. \& Wheeler, W.C. 2005. Systematic review of the frog family Hylidae, with special reference to Hylinae: a phylogenetic analysis and taxonomic revision. Bulletin of the American Museum of Natural History 294:1-240

Fenwick, A., Gutberlet, R. Jr., Evans, J. \& Parkinson, C. 2009. Morphological and molecular evidence for phylogeny and clasification of South American pitvipers, genera Bothrops, Bothriopsis, and Bothrocophias (Serpentes: Viperidae). Zoological Journal of the Linnean Society 156:617-140.

Frost, D.R. 2014. Amphibian Species of the World: an Online Reference. Version 6.0 (23 April 2014). Electronic Database accessible at http://research.amnh.org/ herpetology/amphibia/index.html. American Museum of Natural History, New York, USA.

Grazziotin, F.G., Zaher, H., Murphy, R.W., Scrocchi, G., Benavides, M.A., Zhang, Ya-Ping \& Bonatto, S.L. 2012. Molecular phylogeny of the New World Dipsadidae (Serpentes: Colubroidea): a reappraisal. Cladistics 28(5):437-459.

Hedges, S.B. 2011. The type species of the threadsnake genus Tricheilostoma Jan revisited (Squamata, Leptotyphlopidae). Zootaxa 3027:63-64.

Heyer, W.R. 1994. Variation within the Leptodactylus podicipinuswagneri complex of frogs (Amphibia: Leptodactylidae). Smithsonian Contributions to Zoology 546:1-124.

Heyer, W.R. 2005. Variation and taxonomic clarification of the large species of the Leptodactylus pentadactylus species group (Amphibia: Leptodactylidae) from Middle America, 
Northern South America, and Amazonia. Arquivos de Zoologia 37(3):269-348.

Heyer, R.W. \& De SÁ, R. 2011. Variation, systematics, and relationships of the Leptodactylus bolivianus complex (Amphibia: Anura: Leptodactylidae). Smithsonian Contributions to Zoology 635:1-58

Köhler, G., Batista, A., Vesely, M., Ponce, M., Carrizo, A. \& LotZKat, S. 2012. Evidence for the recognition of two species of Anolis formerly referred to Anolis tropidogaster (Squamata: Dactyloidae). Zootaxa 3348:1-23.

Lampo, M., Señaris, J.C., Rodríguez-Contreras, A., RojasRunJaic, F. \& García, C.Z. 2011. High Turnover Rates in Remnant Populations of the Harlequin Frog Atelopus cruciger (Bufonidae): Low Risk of Extinction? Biotropica 2011:1-7.

La Marca, E. 1994. Taxonomy of the frogs of the genus Mannophryne (Amphibia: Anura: Dendrobatidae). Publicaciones de la Asociación de Amigos de Doñana 4:175.

Lavilla, E.O., Langone, J.A., Padial, J.M. \& de SÁ, R.O. 2010. The identity of the crackling, luminescent frog of Suriname (Rana typhonia Linnaeus, 1758) (Amphibia, Anura). Zootaxa 2671:17-30.

Manzanilla, J., Jowers, J., La Marca, E. \& García-París, M. 2007. Taxonomic reassessment of Mannophryne trinitatis (Anura: Dendrobatidae) with a description of a new species from Venezuela. Herpetological Journal 17:31-42.

Marcuzzi, G. 1950 Breves apuntes sobre algunos lagartos de Venezuela septentrional. Memoria de la Sociedad de Ciencias Naturales La Salle 26:73-110.

Murphy, J.C., Charles, S.P., Lehtinen, R.M. \& Koeller, K.L. 2013. A molecular and morphological characterization of Oliver's Parrot Snake Leptophis caerulodorsus (Squamata: Serpentes: Colubridae) with a description of a new species from Tobago. Zootaxa 3718:561-574.

Narvaes, P. \& Rodrigues, M.T. 2009.Taxonomic revision of
Rhinella granulosa species group (Amphibia, Anura, Bufonidae), with a description of a new species.Arquivos de Zoologia. São Paulo 40:1-73.

Passos, P. \& Fernandes, R. 2008. Revision of the Epicrates cenchria complex (Serpentes: Boidae). Herpetological Monograph 22:1-30.

Peters, J.A. \& Donoso-Barros, R. 1970. Catalogue of the Neotropical Squamata. Part II. Lizards and Amphisbaenians. United States National Museum Bulletin 297:1-293.

Rivas F.G., Ugueto, G., Rivero, R. \& Miralles, A. 2005. The herpetofauna of Isla de Margarita, Venezuela: New records and comments. Caribbean Journal of Science 41:346-351.

Rivas, G., Molina, C.R., Ugueto, G.N., Barros, T.R., BarrioAmorós, C.L. \& KoK, P.J.R. 2012. Reptiles of Venezuela: an updated and commented checklist. Zootaxa 3211:1-64.

Rivero, J.A. 1964. Salientios (Amphibia) en la colección de la Sociedad de Ciencias Naturales La Salle. Caribbean Journal of Science 4(1):297-305.

Rivero, J.A. 1968. Sobre la identidad de Hyla rostrata Peters (Amphibia, Salientia). Acta Biologica Venezuelica 6:133138.

Ruthven, A. 1914. Description of a new species of Basiliscus from the region of the Sierra Nevada de Santa Marta, Colombia. Proceedings of the Biological Society of Washington 27:912.

Savage, J. 2011. The correct species-group name for an Oxyrhopus (Squamata: Dipsadidae) variously called Coluber petalarius, C. pethola, C. petola, or C. petolarius by early authors. Proceedings of the Biological Society of Washington 124:223-225.

Ugueto, G.N. \& Harvey, M.B. 2012. Revision of Ameiva ameiva Linnaeus (Squamata: Teiidae) in Venezuela: recognition of four species and status of introduced populations in southern Florida, USA. Herpetological Monographs 25:113-170.

Recibido: 13.05.14

Aceptado: 30.01 .15 